\title{
Band-gap structure of the spectrum of the water-wave problem in a shallow canal with a periodic family of deep pools
}

\author{
Sergei A. Nazarov ${ }^{1}$. Jari Taskinen ${ }^{2}$
}

Received: 23 July 2020 / Accepted: 22 December 2021 / Published online: 6 January 2022

(C) The Author(s) 2022

\begin{abstract}
We consider the linear water-wave problem in a periodic channel $\Pi^{h} \subset \mathbb{R}^{3}$, which is shallow except for a periodic array of deep potholes in it. Motivated by applications to surface wave propagation phenomena, we study the band-gap structure of the essential spectrum in the linear water-wave system, which includes the spectral Steklov boundary condition posed on the free water surface. We apply methods of asymptotic analysis, where the most involved step is the construction and analysis of an appropriate boundary layer in a neighborhood of the joint of the potholes with the thin part of the channel. Consequently, the existence of a spectral gap for small enough $h$ is proven.
\end{abstract}

Keywords linear water wave equation $\cdot$ periodic channel $\cdot$ shallow channel $\cdot$ spectral gap

\section{Introduction}

\subsection{Formulation of the water-wave problem}

Let $x=(y, z) \in \mathbb{R}^{2} \times \mathbb{R}$ be the Cartesian coordinate system and let $H: \Omega^{0}=$ $\mathbb{R} \times(-\ell, \ell) \rightarrow \mathbb{R}$ be a smooth function, which is periodic in the variable $x_{1}=y_{1}$ for $y=\left(y_{1}, y_{2}\right) \in \Omega^{0}$ as well as positive; precisely, $H(y) \geq H_{0}>0$ in $\overline{\Omega^{0}}$. By rescaling, we reduce the period to one and make all coordinates and geometric

\footnotetext{
$\bowtie \quad$ Jari Taskinen

jari.taskinen@helsinki.fi

Sergei A. Nazarov

srgnazarov@yahoo.co.uk

1 Institute for Problems in Mechanical Engineering of RAS, St. Petersburg 199178, Russia

2 Department of Mathematics and Statistics, University of Helsinki, P.O.Box 68, 00014 Helsinki, Finland
} 

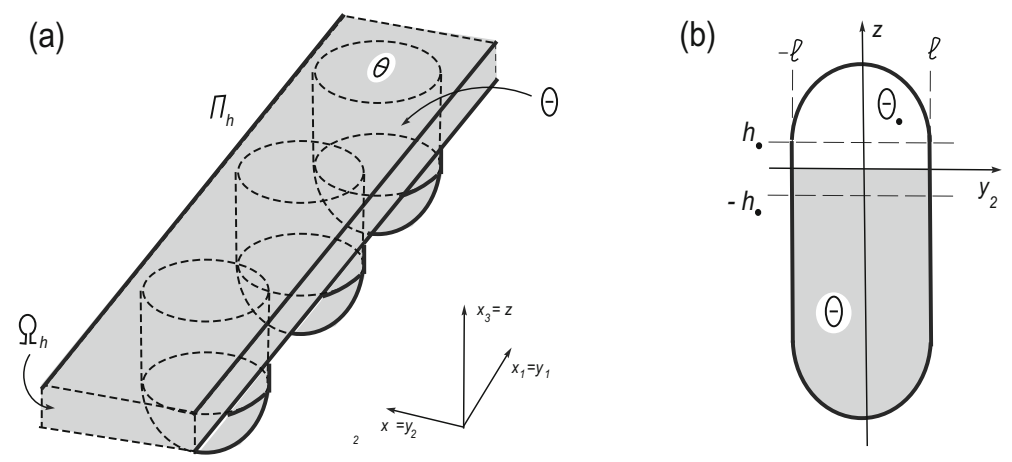

Fig. 1 a Periodic channel, b cross-section of a voluminous container, pothole

parameters dimensionless. We also introduce a domain $\Theta \bullet \subset \mathbb{R}^{3}$, which is contained in the cylinder $(-1 / 2,1 / 2) \times(-\ell, \ell) \times \mathbb{R}$ and has a smooth boundary $\partial \Theta_{\bullet}$ and compact closure $\overline{\Theta_{\bullet}}=\Theta_{\bullet} \cup \partial_{\bullet}$. We assume that the lower part $\Theta=\left\{x \in \Theta_{\bullet}: z<0\right\}$ (Fig. 1b) is non-empty and denote its translates

$$
\Theta_{j}=\left\{x:\left(y_{1}-j, y_{2}, z\right) \in \Theta\right\} \quad \forall j \in \mathbb{Z}=\{0, \pm 1, \pm 2, \ldots\} .
$$

The periodic channel (Fig. 1a)

$$
\Pi^{h}=\Omega^{h} \cup \bigcup_{j \in \mathbb{Z}} \Theta_{j}
$$

consists, in addition to the deep pits (1), of the thin periodic layer

$$
\Omega^{h}=\left\{x=(y, z): 0>z>-h H(y), y \in \Omega^{0}\right\},
$$

where $h>0$ is a small parameter. For the simplicity of the presentation (see Remark 1) we assume that the subdomain $\left\{x \in \Theta_{\bullet}:|z|<h_{\bullet}\right\}$ includes the straight vertical cylinder $\theta \times\left(-h_{\bullet}, h_{\bullet}\right)$, where $h_{\bullet}>0$ is a constant and $\theta=\left\{x \in \Theta_{\bullet}: x_{3}=z=0\right\}$ is the cross-section of $\Theta_{\bullet}$, i.e., the surface $\Upsilon=\left\{x \in \partial \Theta:|z|<h_{\bullet}\right\}$ is cylindrical and perpendicular to the plane $\{x: z=0\}$. Notice that we will not distinguish in the notation between the three-dimensional sets $\Omega^{\circ}, \theta, \partial \theta$ and their embeddings into the above-mentioned horizontal plane.

In the domain $\Pi^{h}$ we consider the water-wave problem, cf. [14], which consists of the Laplace equation for the velocity potential $u^{h}$,

$$
-\Delta u^{h}(x)=0, \quad x \in \Pi^{h},
$$

the Neumann (no penetration) boundary condition on the bottom and walls,

$$
\partial_{\nu} u^{h}(x)=0, \quad x \in \Sigma^{h}=\partial \Pi^{h} \backslash \overline{\Omega^{0}}
$$


and the Steklov (kinematic) spectral condition on the free surface,

$$
\partial_{z} u^{h}(x)=\lambda^{h} u^{h}(x), \quad x=(y, 0) \in \Omega^{0} .
$$

Here, $\Delta$ stands for the Laplacian, $\partial_{v}$ is the outward normal derivative, $\partial_{z}=\partial / \partial z$, and $\lambda^{h}=g^{-1} k_{h}^{2}$ is a spectral parameter, where $g$ is the acceleration of gravity and $k_{h}$ is the physical wave number.

As for the other general notation used in this paper, we write $\mathbb{N}=\{1,2,3, \ldots\}$, $\mathbb{N}_{0}=\{0\} \cup \mathbb{N}$, and $\mathbb{R}_{0}^{+}$for the set of non-negative real numbers. Given a domain $\Xi \subset \mathbb{R}^{d}$, the symbol $|\Xi|$ stands for its volume in $\mathbb{R}^{d}$ and $(\cdot, \cdot)_{\Xi}$ stands for the natural scalar product in Lebesgue space $L^{2}(\Xi)$, and $H^{k}(\Xi), k \in \mathbb{N}$, for the standard Sobolev space of order $k$ on $\Xi$. The norm of a function $f$ belonging to a Banach function space $X$ is denoted by $\|f ; X\|$. For $r>0$ and $a \in \mathbb{R}^{N}, B(a, r)$ (respectively, $S(a, r)$ ) stand for the Euclidean ball (resp. ball surface) with centre $a$ and radius $r$. By $c, C$ (respectively, $c_{k}, C_{k}, c(k)$ etc.) we mean positive constants (resp. constants depending on a parameter $k$ ) which do not depend on functions or variables appearing in the inequalities, but which may still vary from place to place. The gradient and Laplace operators $\nabla$ and $\Delta$ (respectively, in $\nabla_{y}, \Delta_{y}$ etc.) act in the variable $x$ (respectively, $y$ etc.).

\subsection{The goals of the paper}

Our aim is to investigate the band-gap structure of the spectrum of the water-wave problem (4)-(6) and in particular to detect gaps in its essential spectrum. At the end of Sect. 1 we will discuss the standard approach of the Floquet-Bloch-Gelfand-theory, which transforms the problem into a model spectral problem in a bounded domain. The model problem will be treated by the methods of asymptotic analysis in Sect. 2, where the asymptotic ansätze and their essential terms are derived. In Sect. 3, we consider the relationship of the model and limit problems, where the latter corresponds to the case $h=0$. The main result, Theorem 1 contains the crucial estimate, up to corrections of order $O\left(h^{2}(1+|\ln h|)\right)$, between the eigenvalues of the limit problem and those of the model problem (which form the spectral bands of the original problem). Finally, in Sect. 4 we consider the limit problem in more detail under some additional assumptions. We introduce a new geometric parameter $\varepsilon>0$ and observe that, for small enough $\varepsilon$, the limit problem has a spectral gap. The estimate (98) in Theorem 1 then assures that, for small enough $h$, a gap is opened also in the essential spectrum of the problem (4)-(6), although it may be smaller than the one for the limit problem.

Besides the Steklov spectral condition and periodic structure, one more characteristic feature of the problem under consideration is described by junctions of massive bodies with thin ligaments. For example, the dumbbell, which is a union of two massive domains connected by a thin cylinder, is a classical object in asymptotic analysis, and the spectral Laplace-Neumann problem and asymptotic expansions for eigenvalues and eigenfunctions have been considered in many papers. Concerning asymptotic methods, we will here partly follow the approach in [21] and [22], although the aims in these references are different as they include topics like self-adjoint extension of the 
problem operator and eigenvalue estimates also in the high-frequency range, instead of spectral gaps corresponding to low frequencies in the present work. We mention the paper [7], where the two-dimensional version of the present problem was treated with result on the existence of arbitrarily many spectral gaps for thin enough (small $h>0$ ) connecting canals of voluminous containers. However, we emphasize that in the lower dimensional case the limit problem is an ordinary differential equation, the solutions of which can be found explicitly and quite precise information of its spectrum, including spectral gaps, can be obtained by a quite elementary approach. Here, the limit problem is still a spectral Laplace problem, and precise enough information on its spectrum is more difficult to gain. Also, it is clear that the structure of the asymptotic terms is essentially different and more complicated in the case $d=3$.

Of course, neither the present paper nor [7] are the first studies of spectral gaps by means of asymptotic analysis. Let us mention [3,4,6,20,23], where the detection of open gaps is based on a periodic perforation of strips and cylinders or singular perturbations of a similar type, an approach which will also be used in Sect. 4.

\subsection{Model problem in the periodicity cell}

Our approach to the problem (4)-(6) is based on the Floquet-Bloch-Gelfand- (FBG)transform, which converts the problem on the periodic channel $\Pi^{h}$ into one on the periodicity cell

$$
\varpi^{h}=\left\{x \in \Pi^{h}:\left|y_{1}\right|<1 / 2\right\} .
$$

The boundary $\partial \varpi^{h}$ includes the free water surface $\omega^{0}=\left\{x \in \Omega^{0}:\left|y_{1}\right|<1 / 2\right\}$ and the lateral surface $\varsigma^{h}=\left\{x \in \Sigma^{h}:\left|y_{1}\right|<1 / 2\right\}$. We recall that the FBG-transform is defined by

$$
u(x) \mapsto U(x, \eta)=\frac{1}{\sqrt{2 \pi}} \sum_{j \in \mathbb{Z}} e^{-i \eta j} u\left(x_{1}+j, x_{2}, x_{3}\right)
$$

where $x=\left(x_{1}, x_{2}, x_{3}\right) \in \Pi^{h}$ on the left while, on the right, we have $x \in \varpi^{h}$ and $\eta \in[-\pi, \pi]$; this is the dual variable or the Floquet parameter. For more details, see [9] and e.g. [27, XII.16], [24, § 3.4], [18, Cor. 3.4.3], [13, Sec.2.2]. Applying the FBG-transform to the problem (4)-(6), we obtain a family of model problems in the periodicity cell $\varpi^{h}$ parametrized by $\eta$,

$$
\begin{aligned}
-\Delta U^{h}(\eta ; x) & =0, \quad x \in \varpi^{h}, \\
\partial_{\nu} U^{h}(\eta ; x) & =0, \quad x \in \varsigma^{h}, \\
\partial_{z} U^{h}(\eta ; x) & =\Lambda^{h}(\eta) U^{h}(\eta ; x), \quad x \in \omega^{0}, \\
\left.U^{h}(\eta ; x)\right|_{y_{1}=1 / 2} & =\left.e^{i \eta} U^{h}(\eta ; x)\right|_{y_{1}=-1 / 2} \\
\left.\frac{\partial U^{h}}{\partial x_{1}}(\eta ; x)\right|_{y_{1}=1 / 2} & =\left.e^{i \eta} \frac{\partial U^{h}}{\partial x_{1}}(\eta ; x)\right|_{y_{1}=-1 / 2}
\end{aligned}
$$


Here, $\Lambda=\Lambda(h, \eta)$ is a new notation for the spectral parameter $\lambda$.

As is well known, the FBG-transform establishes an isometric isomorphism

$$
L^{2}\left(\Pi^{h}\right) \simeq L^{2}\left(0,2 \pi ; L^{2}\left(\varpi^{h}\right)\right)
$$

where $L^{2}(0,2 \pi ; B)$ is the Lebesgue space of functions with values in the Banach space $B$, endowed with the norm

$$
\left\|f ; L^{2}(0,2 \pi ; B)\right\|=\left(\int_{0}^{2 \pi}\|f(\eta) ; B\|^{2} d \eta\right)^{1 / 2}
$$

We denote by $H_{\eta}^{1}\left(\varpi^{h}\right)$, where $\eta \in[0,2 \pi)$, the subspace of the Sobolev space $H^{1}\left(\varpi^{h}\right)$ consisting of functions satisfying the quasiperiodic boundary condition (11). The FBG-transform is also an isomorphism from the Sobolev space $H^{1}\left(\Pi^{h}\right)$ onto $H^{1}\left(0,2 \pi ; H_{\eta}^{1}\left(\varpi^{h}\right)\right)$; see the references above.

Our approach to the spectral properties of the model is similar to [21,25] and others. Given $\eta \in[-\pi, \pi]$, we write the variational formulation of the problem (8)-(12) for the unknown function $U \in H_{\eta}^{1}\left(\Omega^{h}\right)$ and the spectral parameter $\Lambda(\eta)$ as

$$
\begin{aligned}
& \left(\nabla U^{h}(\eta ; \cdot), \nabla \psi^{h}(\eta ; \cdot)\right)_{\varpi^{h}} \\
& =\Lambda^{h}(\eta)\left(U^{h}(\eta ; \cdot), \psi^{h}(\eta ; \cdot)\right)_{\omega^{0}}, \forall \psi^{h}(\eta ; \cdot) \in H_{\eta}^{1}\left(\varpi^{h}\right) .
\end{aligned}
$$

We denote in the sequel by $\mathcal{H}_{\eta}^{h}$ the space $H_{\eta}^{1}\left(\varpi^{h}\right)$ endowed with the new scalar product

$$
\left\langle u^{h}, \psi^{h}\right\rangle_{h}=\left(\nabla u^{h}, \nabla \psi^{h}\right)_{\varpi^{h}}+h\left(u^{h}, \psi^{h}\right)_{\omega^{0}},
$$

where $\eta$ is omitted from the notation and the inner product in $\omega^{0}$ is understood in the sense of traces, and define a self-adjoint, positive operator $\mathcal{K}_{\eta}^{h}: \mathcal{H}_{\eta}^{h} \rightarrow \mathcal{H}_{\eta}^{h}$ by using the identity

$$
\left\langle\mathcal{K}_{\eta}^{h} u^{h}, \psi^{h}\right\rangle_{h}=\left(u^{h}, \psi^{h}\right)_{\omega^{0}} \forall u^{h}, \psi^{h} \in \mathcal{H}_{\eta}^{h}
$$

The operator $\mathcal{K}_{\eta}^{h}$ is also compact due to the compactness of the embedding $H^{1}\left(\varpi^{h}\right) \subset$ $L^{2}\left(\omega^{0}\right)$. The integral identity (13) corresponding to the problem (8)-(12) is then equivalent with the standard spectral equation in the Hilbert space $\mathcal{H}_{\eta}^{h}$,

$$
\mathcal{K}_{\eta}^{h} U^{h}=\kappa^{h} U^{h}
$$

with the new spectral parameter

$$
\kappa^{h}=\kappa^{h}(\eta)=\left(\Lambda^{h}(\eta)+h\right)^{-1}
$$


(a)

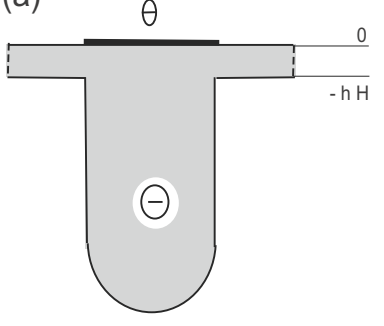

(b)

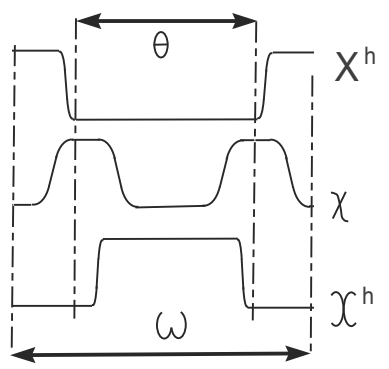

Fig. 2 a Cross-section of the structure of a pothole, $\mathbf{b}$ graphs of cut-off functions

According to $\left[5\right.$, Thm. 10.1.5, 10.2.2] the spectrum of $\mathcal{K}_{\eta}^{h}$ consists of null, which is the only point in the essential spectrum, and a positive sequence of eigenvalues belonging to the discrete spectrum

$$
\kappa_{1}^{h} \geq \kappa_{2}^{h} \geq \ldots \geq \kappa_{m}^{h} \geq \ldots \rightarrow+0
$$

and, owing to (15) and (14), the norm of the operator $\mathcal{K}_{\eta}^{h}$ does not exceed $h^{-1}$ so that

$$
\kappa_{1}^{h} \leq 1
$$

Formula (16) and the properties of the sequence $\left(\kappa_{k}^{h}(\eta)\right)_{k=1}^{\infty}$ mean that the eigenvalues of the problem (8)-(12) form an unbounded sequence

$$
0 \leq \Lambda_{1}^{h}(\eta) \leq \Lambda_{2}^{h}(\eta) \leq \ldots \leq \Lambda_{k}^{h}(\eta) \leq \ldots \rightarrow+\infty
$$

where multiplicities have been taken into account. Note that the first inequality in (19) follows from (18) and (16) but also directly from the integral identity (13). We denote for every $k$ by $U_{k}^{h}(\eta ; \cdot) \in H_{\eta}^{1}\left(\varpi^{h}\right)$ the eigenfunction corresponding to $\Lambda_{k}^{h}(\eta)$, which fulfil the normalization and orthogonality conditions

$$
\left(U_{m}^{h}(\eta ; \cdot), U_{n}^{h}(\eta ; \cdot)\right)_{\omega^{0}}=\delta_{m, n}, \quad m, n \in \mathbb{N},
$$

where on the right-hand side there is the Kronecker delta.

Finally, the functions $\eta \mapsto \Lambda_{k}^{h}(\eta)$ are continuous and $2 \pi$-periodic (see for example [11, Ch.9], [13, Sec.3.1]). Hence, the spectral bands $B_{m}^{h}=\left\{\Lambda_{m}^{h}(\eta): \eta \in[0,2 \pi)\right\}$ of the problem (4)-(6) indeed are compact intervals, and, by well-known principles of the FBG-theory, the essential spectrum $S_{\mathrm{ess}}^{h}$ of the problem (4)-(6) can be presented as

$$
S_{\mathrm{ess}}^{h}=\bigcup_{m \in \mathbb{N}} B_{m}^{h}
$$




\section{Formal asymptotic analysis}

\subsection{Asymptotic ansätze}

We search for an eigenvalue of the problem (8)-(12) in the form

$$
\Lambda^{h}(\eta)=h \mu(\eta)+\ldots
$$

Here, and later in this section, dots stand for higher order terms, which are inessential for our preliminary analysis and will eventually be estimated in Theorem 1. Inside the thin part $\varpi_{\sharp}^{h}=\varpi^{h} \backslash \bar{\Theta}$ of the periodicity cell, we employ the standard asymptotic decomposition in shallow water domains

$$
U^{h}(\eta ; x)=v^{0}(\eta ; y)+h^{2} v^{\prime}(\eta ; y, \zeta)+\ldots \quad \text { in } \varpi_{\sharp}^{h},
$$

cf. $[7,8,21,22]$ and others. Here, $\zeta=h^{-1} z$ is the stretched vertical coordinate. We note that

$$
\partial_{v}=\left(1+h^{2}\left|\nabla_{y} H(y)\right|^{2}\right)^{-1 / 2}\left(-\partial_{z}-h \nabla_{y} H(y) \cdot \nabla_{y}\right)
$$

at the bottom part $\varsigma_{\sharp}^{h}=\left\{x \in \omega^{0} \backslash \bar{\theta}: z=-h H(y)\right\}$ and insert the ansätze into the Eq. (8), restricted to $\varpi_{\sharp}^{h}$, and to the boundary conditions (9) and (10), restricted to $\varsigma_{\sharp}^{h}$ and $\omega_{\sharp}^{0}=\omega^{0} \backslash \bar{\theta}$, respectively. Separating terms of the same order in $h$ yields the problems

$$
\begin{aligned}
-\partial_{\zeta}^{2} v^{0}(\eta ; y) & =0 \text { for } \zeta \in(-H(y), 0), \\
\partial_{\zeta} v^{0}(\eta ; y) & =0 \text { for } \zeta=-H(y) \text { and } \zeta=0
\end{aligned}
$$

(which trivially holds, since $v$ is independent of $\zeta$ in (22)), and

$$
\begin{aligned}
& -\partial_{\zeta}^{2} v^{\prime}(\eta ; y, \zeta)=\Delta_{y} v^{0}(\eta ; y), \zeta \in(-H(y), 0), \\
& -\partial_{\zeta} v^{\prime}(\eta ; y,-H(y))=\nabla_{y} H(y) \cdot \nabla_{y} v^{0}(\eta ; y),-\partial_{\zeta} v^{\prime}(\eta ; y, 0)=\mu v^{0}(\eta ; y) .
\end{aligned}
$$

Equations (24) form a Neumann problem for an ordinary differential equation, and it has a solution, if and only if the compatibility condition

$$
\int_{-H(y)}^{0} \Delta_{y} v^{0}(\eta ; y) d \zeta+\nabla_{y} H(y) \cdot \nabla_{y} v^{0}(\eta ; y)+\mu(\eta) v^{0}(\eta ; y)=0
$$

is satisfied. This can be written as the differential equation

$$
-\nabla_{y} \cdot H(y) \nabla_{y} v^{0}(\eta ; y)=\mu(\eta) v^{0}(\eta ; y), \quad y \in \omega_{\sharp}^{0} .
$$


Due to the form of the ansatz (22), the conditions (9), (11) and (12) lead to the following boundary and quasiperiodicity conditions

$$
\begin{aligned}
& \pm \frac{\partial v^{0}}{\partial y_{2}}\left(\eta ; y_{1}, \pm \ell\right)=0, \quad\left|y_{1}\right|<\frac{1}{2}, \\
& v^{0}\left(\eta ; \frac{1}{2}, y_{2}\right)=e^{i \eta} v^{0}\left(\eta ;-\frac{1}{2}, y_{2}\right), \quad\left|y_{2}\right|<\ell, \\
& \frac{\partial v^{0}}{\partial y_{1}}\left(\eta ; \frac{1}{2}, y_{2}\right)=e^{i \eta} \frac{\partial v^{0}}{\partial y_{1}}\left(\eta ;-\frac{1}{2}, y_{2}\right), \quad\left|y_{2}\right|<\ell .
\end{aligned}
$$

It remains to derive a condition on the interior boundary $\partial \theta$ of the domain $\omega_{\sharp}^{h} \subset \mathbb{R}^{2}$. To this end we use the asymptotic ansatz, cf. [7,21,22],

$$
U^{h}(\eta ; x)=V^{0}(\eta ; x)+h V^{\prime}(\eta ; x)+\ldots
$$

in the massive part $\Theta$ of the periodicity cell (7). We insert the ansätze (29) and (21) into the Eq. (8) in $\Theta$, into the Steklov condition (10) in $\theta$ and into the Neumann condition (9) on the surface $\varsigma^{h} \cap \partial \Theta$. The curved two-dimensional ring

$$
v^{h}=\{x: y \in \partial \theta,-h H(y)<z<0\}
$$

does not appear in the boundary condition (9) and it also disappears on the limit $h \rightarrow+0$ so that it is natural to compose the problems

$$
-\Delta V^{0}(\eta ; x)=0 \text { for } x \in \Theta, \quad \partial_{\nu} V^{0}(\eta ; x)=0 \text { for } x \in \partial \Theta \backslash \partial \theta,
$$

and

$$
\begin{aligned}
& -\Delta V^{\prime}(\eta ; x)=0 \text { for } x \in \Theta, \quad \partial_{\nu} V^{\prime}(\eta ; x)=0 \text { for } x \in \partial \Theta \backslash \bar{\theta}, \\
& -\partial_{z} V^{\prime}(\eta ; x)=\mu(\eta) V^{0}(\eta ; x), \quad x \in \theta .
\end{aligned}
$$

According to (31), the main asymptotic term in (29) is a constant,

$$
V^{0}(\eta ; x)=a^{0}(\eta)
$$

however, the Neumann problem (32) has no bounded solution, if $\mu(\eta) \neq 0$ and $a^{0}(\eta) \neq 0$. This leads to the conclusion that the problem (32) has to be modified [remember how we treated the ring (30)].

\subsection{Boundary layer}

We will link the outer expansion (22) and (29) by using the method of matched asymptotic expansions, see the monographs [10,28] and the papers [7,21,22], where applications to the water-wave problem are considered. To construct the inner expansion in the vicinity of $v^{h}$, we introduce the local curvilinear coordinates $(s, n, z)$ in a 

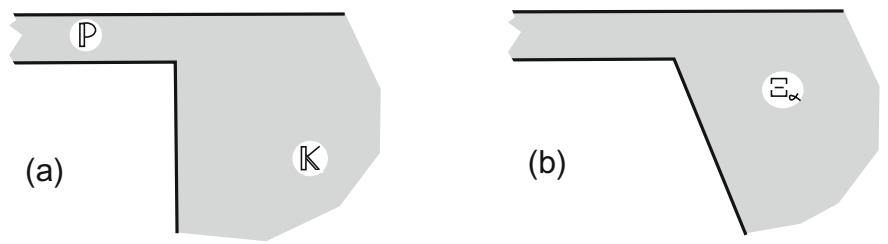

Fig. 3 a The scaled domain $\Xi=\mathbb{K} \cup \mathbb{P}$, b the domains $\Xi_{\alpha}$ in the non-perpendicular case

three-dimensional neighborhood $\mathcal{V}$ of the contour $\partial \theta$, such that $s$ is the arc length on $\partial \theta, n$ is the oriented distance to $\partial \theta$ on $\omega^{0}$ with $n>0$ inside $\theta$, and $z=x_{3}$. We keep $s$ unscaled but change $n$ and $z$ into the stretched coordinates

$$
\xi=\left(\xi_{1}, \xi_{2}\right)=\frac{1}{h} \frac{1}{H(s)}(n, z) \text { with } H(s)=\left.H(y)\right|_{\partial \theta} .
$$

The coordinate change $x \mapsto(s, \xi)$ and the formal replacement $h=0$ transform the subdomain $\varpi^{h} \cap \mathcal{V}$ into the set

$$
\{(s, \xi): s \in \partial \theta, \xi \in \Xi\}
$$

where $\Xi$ is the union of the fourth quadrant of the plane, $\mathbb{K}=\left\{\xi \in \mathbb{R}^{2}: \xi_{1}>0, \xi_{2}<\right.$ $0\}$ and the semi-infinite strip $\mathbb{P}=\left\{\xi \in \mathbb{R}^{2}: \xi_{1} \leq 0, \xi_{2} \in(-1,0)\right\}$ of unit width [recall the denominator $H(s)$ in (34); see Fig. 3a].

We have $\partial_{v}=\partial_{n}=h^{-1} \partial / \partial \xi_{1}$ for $y \in \partial \theta, z \in\left(-h_{\bullet}, 0\right)$ with $h_{\bullet}>0$ defined in Sect. 1.1 and $\partial_{z}=h^{-1} \partial / \partial \xi_{2}$ on $\omega^{0}$. The Laplacian $\Delta_{x}$ is written in the curvilinear coordinates as

$$
(1+n \varkappa(s))^{-1}\left(\frac{\partial}{\partial s}(1+n \varkappa(s)) \frac{\partial}{\partial s}+\frac{\partial}{\partial n}(1+n \varkappa(s))^{-1} \frac{\partial}{\partial n}\right)+\frac{\partial^{2}}{\partial z^{2}},
$$

where $\varkappa(s)$ is the curvature of the contour $\partial \theta \ni s$. Thus, the coordinate change $x \mapsto \xi$ together with formulas (35) and (23) and passing formally to the limit $h \rightarrow+0$ turn (8)-(10) into the Neumann problem

$$
-\Delta_{\xi} w(\xi)=0, \quad \xi \in \Xi, \quad \partial_{\nu(\xi)} w(\xi)=0, \quad \xi \in \partial \Xi \backslash P,
$$

where $P=(0,-1)$; this is the only corner point with opening $\alpha=3 \pi / 2$ on the boundary $\partial \Xi$.

Remark 1 If the surface $\partial \Theta$ is not perpendicular to the plane $\{x: z=0\}$ on the contour $\partial \theta$, then, instead of the union $\Xi_{3 \pi / 2}=\mathbb{K} \cup \mathbb{P}$ (Fig. 3b) we obtain the domain

$$
\Xi_{\alpha(s)}=\mathbb{K}_{\alpha(s)} \cup\left\{\xi: \xi_{1} \in \mathbb{R}, \xi_{2} \in(-1,0)\right\},
$$

where $\mathbb{K}_{\alpha(s)}=\left\{\xi: \xi_{2}<0, \xi_{1}>-\xi_{2} \cot (\alpha(s))\right\}$ is the angle of opening $\alpha(s) \in$ $(\pi, 2 \pi)$. In this case the formal asymptotic analysis would remain almost the same, 
but the justification procedure of Sect. 3 would become more involved with more cumbersome calculations.

To follow the matching procedure in [7,21], we need to find the solutions of the problem (36) with linear behavior at the infinity in $\mathbb{P}$. One of them is obvious, the constant function. However, there also exists a harmonic function $W$ in $\Xi$ satisfying the Neumann condition such that

$$
W(\xi)= \begin{cases}\xi_{1}+c_{\Xi}+O\left(e^{\pi \xi_{1}}\right), & \xi_{1} \rightarrow-\infty, \quad \xi \in \mathbb{P}, \\ 2 \pi^{-1} \ln |\xi|+O\left(|\xi|^{-1}\right), & \left|\xi_{1}\right| \rightarrow+\infty, \quad \xi \in \mathbb{K},\end{cases}
$$

see for example $[22, \S 2]$. Here $c_{\Xi}$ is an absolute constant which can be found by solving the problem by an appropriate conformal mapping, but we do not need the exact value in the following. Note that the solution is made unique by the requirement that the constant term of the solution, which remains undetermined by the Neumann condition, equals 0 in the quadrant $\mathbb{K}$.

Furthermore, it follows from the Kondratiev theory (see [12] and, e.g., [24, Ch. 2]) that all solutions of (36) with at most polynomial growth rate in $\mathbb{P}$ and logarithmic in $\mathbb{K}$ are given by the linear combinations

$$
c_{0}+c_{1} W(\xi), \quad c_{0}, c_{1} \in \mathbb{R}
$$

Let us now realize the matching procedure. The Taylor formula in the variable $n$ converts the outer expansion (22) in the thin domain $\varpi_{\sharp}^{h}$ into

$$
\begin{aligned}
U^{h}(\eta ; x) & =v(\eta ; s, 0)+n \partial_{n} v(\eta ; s, 0)+\cdots+h^{2} v^{\prime}(\eta ; s, 0)+\cdots \\
& =v(\eta ; s, 0)+h H(s) \partial_{n} v(\eta ; s, 0)+\cdots
\end{aligned}
$$

where $v(\eta ; s, n)$ is the function $v(\eta ; \cdot)$ written in the curvilinear coordinates. Comparing terms of order $1=h^{0}$ in the outer expansion (38) and (29) with the inner expansions

$$
U^{h}(x)=c^{0}(\eta ; s)+h c^{1}(\eta ; s) W\left(h^{-1} H(s)^{-1} n,\left(h^{-1} H(s)^{-1} z\right)+\ldots\right.
$$

we conclude that

$$
v^{0}(\eta ; s, 0)=c^{0}(\eta ; s)=a^{0}(\eta)
$$

where $a^{0}(\eta)$ is taken from (33).

To continue, we compare (38) and (39) at the level $h$. The asymptotics (37) in $\mathbb{P}$ requires that

$$
c^{1}(\eta ; s)=H(s) \partial_{n} v^{0}(\eta ; s, 0) .
$$

Hence, in view of the representation (37) in $\mathbb{K}$, the correction term of the outer expansion (29) in the massive part $\Theta$ of the periodicity cell $\varpi^{h}$ must have the following 
behavior at edge $\partial \theta$ of the boundary $\partial \Theta$ :

$$
V^{\prime}(\eta ; x)=\frac{2}{\pi} H(s) \partial_{n} v^{0}(\eta ; s, 0) \ln r+O(1) \text { as } r=\left(n^{2}+z^{2}\right)^{1 / 2} \rightarrow+0 .
$$

It should be mentioned that the thin ring (30) does not involve any boundary condition and it shrinks into the edge $\partial \theta$ of the container $\Theta$ so that it is not possible to make a priori any conclusion on the behavior of $V^{\prime}(\eta ; x)$ as $x$ approaches $\partial \theta$. At the same time, the matching procedure leads to the relation (41), which completes the formulation of the Neumann problem for $V^{\prime}$.

A solution of the problem (32), (41) can be found in the form

$$
V^{\prime}(\eta ; x)=\chi(x) \frac{2}{\pi} H(s) \partial_{n} v^{0}(\eta ; s, 0) \ln r+\widehat{V}^{\prime}(\eta ; x),
$$

where $\chi$ is a smooth cut-off function which is equal to 1 in the vicinity of $\partial \theta$ and has support in $\bar{\Theta} \cap \mathcal{V}$. The function $\widehat{V}^{\prime}(\eta, x)$ can be found from the Poisson equation with the right-hand side $\widehat{f}$ such that $r^{\delta} \widehat{f} \in L^{2}(\Theta)$ and with Neumann data in $L^{2}(\partial \theta)$. Here, $r(x)=\operatorname{dist}(x, \partial \theta)$ and $\delta>0$ is arbitrary. Hence, the existence of $\widehat{V}^{\prime} \in H^{1}(\Theta)$ follows by posing one compatibility condition. This can of course be derived from the original problem (32), (41) by integrating by parts in the truncated domain $\Theta(\varrho)=$ $\{x \in \Theta: r(x)>\varrho\}$ and sending $\varrho$ to +0 . Indeed,

$$
\begin{aligned}
\mu \operatorname{mes}_{2}(\theta) a^{0}(\eta) & =\lim _{\varrho \rightarrow+0} \int_{\{y \in \theta: r>\varrho\}} \partial_{z} V^{\prime}(\eta ; y, 0) d y \\
& =\lim _{\varrho \rightarrow+0} \int_{\{x \in \Theta \cap \mathcal{V}: r=\varrho\}} \partial_{r} V^{\prime}(\eta ; x) d s_{x} \\
& =\int_{\partial \theta} H(s) \partial_{n} v^{0}(\eta ; s, 0) d s \frac{2}{\pi} \int_{0}^{\pi / 2} d \varphi=\int_{\partial \theta} H(s) \partial_{n} v^{0}(\eta ; s, 0) d s,
\end{aligned}
$$

where $\varphi$ is the angular variable of the polar coordinate system $(r, \varphi)$ in planes perpendicular to the contour $\partial \theta$.

As a result of our calculations, we conclude by associating the following conditions on $\partial \theta$ to the problems (25)-(28),

$$
\begin{aligned}
& v^{0}(\eta ; s, 0)=a^{0}(\eta), \quad s \in \partial \theta \\
& \int_{\partial \theta} H(s) \partial_{n} v^{0}(\eta ; s, 0) d s=\mu(\eta) a^{0}(\eta) \operatorname{mes}_{2}(\theta) .
\end{aligned}
$$

The quantity $a^{0}(\eta)$ has not been fixed yet, but it must be found by solving the obtained eigenvalue problem (25)-(28), (42), which we call the limit problem. 


\subsection{The spectrum of the limit problem}

To present the variational formulation of the problem (25)-(28), (42) we introduce the following subspace of the Sobolev space $H^{1}\left(\omega_{\sharp}^{0}\right)$ :

$$
\mathcal{H}(\eta)=\left\{v^{0} \in H^{1}\left(\omega_{\sharp}^{0}\right): v^{0} \text { satisfies (27) and is constant on } \partial \theta \subset \partial \omega_{\sharp}^{0}\right\} \text {. }
$$

Notice that the space (43) involves only the stable periodicity condition (27), while the other condition (28) is the intrinsic one in the terminology of [15].

We denote the a priori unknown constant in (43) by $v_{\theta}^{0} \in \mathbb{C}$. Owing to (42) and (43) we have

$$
\int_{\partial \theta} H(s) v^{0}(\eta ; s, 0) \overline{\psi(\eta ; s, 0)} d s=\mu(\eta) v_{\theta}^{0} \overline{\psi_{\theta}} \operatorname{mes}_{2}(\theta)
$$

for any solution $v^{0} \in H^{2}\left(\omega_{\sharp}^{0}\right)$ of the limit problem and test function $\psi(\eta ; \cdot) \in \mathcal{H}(\eta)$, hence, multiplying $(25)$ by $\overline{\psi(\eta ; x)}$, integrating by parts, and taking into account the boundary and quasiperiodicity conditions (25)-(28) yield the integral identity

$$
\begin{aligned}
& \left(H \nabla_{y} v^{0}(\eta ; \cdot), \nabla_{y} \psi(\eta ; \cdot)\right)_{\omega_{\sharp}^{0}} \\
= & \mu(\eta)\left(\left(v^{0}(\eta ; \cdot), \psi(\eta ; \cdot)\right)_{\omega_{\sharp}^{0}}+v_{\theta}^{0}(\eta) \overline{\psi_{\theta}(\eta)} \operatorname{mes}_{2}(\theta) \quad \forall \psi(\eta ; \cdot) \in \mathcal{H}(\eta),\right.
\end{aligned}
$$

where $(\cdot, \cdot) \omega_{\sharp}^{0}$ is the natural scalar product of the Lebesgue space $L^{2}\left(\omega_{\sharp}^{0}\right)$.

Since the embeddings of $H^{1}\left(\omega_{\sharp}^{0}\right)$ into $L^{2}\left(\omega_{\sharp}^{0}\right)$ and $L^{2}(\partial \theta)$ are compact, we observe that (43) is a closed subspace of $H^{1}\left(\omega_{\sharp}^{0}\right)$. Moreover, the sesquilinear form on left-hand side of (44) is positive, thus Hermitian, and closed in $H^{1}\left(\omega_{\sharp}^{0}\right)$. These observations obviously imply the following assertion.

Proposition 1 The spectrum of the problem (44) is discrete and it consists of the positive monotone unbounded sequence of eigenvalues

$$
0 \leq \mu_{1}(\eta) \leq \mu_{2}(\eta) \leq \ldots \leq \mu_{m}(\eta) \leq \ldots \rightarrow+\infty
$$

where multiplicities are taken into account. The corresponding eigenfunctions $v_{1}^{0}(\eta ; \cdot)$, $v_{2}^{0}(\eta ; \cdot), \ldots, v_{m}^{0}(\eta ; \cdot), \ldots \in \mathcal{H}(\eta)$ can be subject to normalization and orthogonality conditions

$$
\left(v_{m}^{0}(\eta ; \cdot), v_{n}^{0}(\eta ; \cdot)\right)_{\omega_{\sharp}^{0}}+v_{m \theta}^{0}(\eta) \overline{v_{n \theta}^{0}(\eta)} \operatorname{mes}_{2}(\theta)=\delta_{m, n} \quad m, n \in \mathbb{N} .
$$

Since the boundary $\partial \theta$ was assumed smooth, also the eigenfunctions $v_{m}^{0}(\eta, \cdot)$ are smooth, see $[1,2]$, and therefore the eigenpairs $\left\{\mu_{m}(\eta), v_{m}^{0}(\eta, \cdot)\right\}$ of the variational problem (44) are solutions of the differential problem (25)-(28), (31), too. 
The first eigenvalue and eigenfunction of the limit problem satisfy the relations

$$
0=\mu_{1}(0)<\mu_{1}(\eta) \text { for } \eta \in[-\pi, 0) \cup(0, \pi], \quad v_{1}^{0}(0 ; y)=(2 \ell)^{-1 / 2} .
$$

It is remarkable that the first eigenpair $\left\{\Lambda_{1}^{h}(0), U_{1}^{h}(0, x)\right\}$ of the original problem (8)(12) with $\eta=0$ has the same form $\left\{0,(2 \ell)^{-1 / 2}\right\}$.

\section{Justification of asymptotics}

\subsection{Convergence theorem}

We consider an eigenpair $\left\{\Lambda_{n}^{h}(\eta), U_{n}^{h}(\eta ; \cdot)\right\}$ of the problem (13) with a fixed number $n \in \mathbb{N}$, where $U_{n}^{h}(\eta ; \cdot)$ is normalized as in (20) and

$$
\Lambda_{n}^{h}(\eta) \leq C^{(n)} h \text { for } h \in\left(0, h^{(n)}\right]
$$

for some positive $h^{(n)}$ and $C^{(n)}$; the bound (47) will be derived in Remark 2 in Sect. 3.4. Hence, there exists a positive sequence $\left\{h_{j}\right\}_{j \in \mathbb{N}}$ tending to 0 such that

$$
h_{j}^{-1} \Lambda_{n}^{h_{j}}(\eta) \rightarrow \widehat{\mu}_{n}(\eta) \in[0,+\infty)
$$

In what follows we omit the argument $h$ and the index $n$ from the notation and also write simply $h$ instead of $h_{j}$.

We pick up a function $\psi \in C^{\infty}\left(\overline{\omega_{\sharp}^{0}}\right)$ which satisfies the first quasiperiodicity condition (27) and coincides with $\psi_{\theta} \in \overleftrightarrow{\mathbb{C}}$ on $\partial \theta$. We insert the special test function

$$
\psi^{h}(x)= \begin{cases}\psi(y), & x \in \varpi_{\sharp}^{h}, \\ \psi_{\theta}, & x \in \Theta,\end{cases}
$$

into the integral identity (13) and rewrite it as follows:

$$
\begin{aligned}
& h^{-1} \int_{\varpi_{\sharp}^{h}} \nabla_{y} U^{h}(x) \cdot \overline{\nabla_{y} \psi(y)} d x-h^{-1} \Lambda^{h} \int_{\omega_{\sharp}^{0}} U^{h}(y, 0) \overline{\psi(y)} d y \\
= & h^{-1} \Lambda^{h} \int_{\theta} U^{h}(y, 0) \overline{\psi_{\theta}} d y .
\end{aligned}
$$

We set $\Psi^{h}=U^{h}$ in (13) and use (47), (20) to derive the estimate

$$
\left\|\nabla U^{h} ; L^{2}\left(\varpi^{h}\right)\right\|^{2}=\Lambda^{h}\left\|U^{h} ; L^{2}\left(\omega^{0}\right)\right\|^{2}=\Lambda^{h} \leq c h
$$


so that the Poincaré and trace inequalities in the massive part $\Theta$ yield

$$
\begin{aligned}
& U^{h}(x)=U_{0}^{h}+U_{\perp}^{h}(x), \quad U_{0}^{h} \in \mathbb{C}, \int_{\Theta} U_{\perp}^{h}(x) d x=0, \\
& \left\|U_{\perp}^{h} ; L^{2}(\Theta)\right\|^{2} \leq c\left\|\nabla U_{\perp}^{h} ; L^{2}(\Theta)\right\|^{2}=c\left\|\nabla U^{h} ; L^{2}(\Theta)\right\|^{2} \\
& \leq c \Lambda^{h}\left\|U^{h} ; L^{2}\left(\omega^{0}\right)\right\|^{2} \leq c h, \\
& \left\|U_{\perp}^{h} ; L^{2}(\theta)\right\|^{2} \leq c\left\|U_{\perp}^{h} ; H^{1}(\Theta)\right\|^{2} \leq c h .
\end{aligned}
$$

Hence, we can pass to a subsequence of $\left\{h_{j}\right\}_{j \in \mathbb{N}}$, and still keep the above convention on the notation, so that

$$
\left.U^{h}\right|_{z=0} \rightarrow \widehat{v}_{\theta} \in \mathbb{C} \quad \text { strongly in } L^{2}(\theta)
$$

Therefore we obtain for the right-hand side $I_{\mathrm{ri}}^{h}(\psi)$ of (49)

$$
I_{\mathrm{ri}}^{h}(\psi) \rightarrow \widehat{\mu} \operatorname{mes}_{2}(\theta) \widehat{v}_{\theta} \overline{\psi_{\theta}} .
$$

To process the left-hand side $I_{\mathrm{le}}^{h}(\psi)$ of (49) we define the stretched domain $\varpi_{\sharp}^{1}=$ $\left\{(y, \zeta): y \in \omega_{\sharp}^{0},-H(y)<\zeta<0\right\}$ and observe that the function $\mathbf{U}^{h}(y, \zeta)=$ $U^{h}\left(y, h^{-1} z\right)$ satisfies

$$
\begin{aligned}
& \left\|\mathbf{U}^{h}(\cdot, 0) ; L^{2}\left(\omega_{\sharp}^{0}\right)\right\|^{2}+\left\|\nabla_{y} \mathbf{U}^{h}(\cdot, 0) ; L^{2}\left(\varpi_{\sharp}^{1}\right)\right\|^{2}+h^{-2}\left\|\partial_{\zeta} \mathbf{U}^{h}(\cdot, 0) ; L^{2}\left(\varpi_{\sharp}^{1}\right)\right\|^{2} \\
& \quad=\left\|U^{h}(\cdot, 0) ; L^{2}\left(\omega_{\sharp}^{0}\right)\right\|^{2}+h^{-1}\left\|\nabla_{y} U^{h} ; L^{2}\left(\varpi_{\sharp}^{h}\right)\right\|^{2}+h^{-1}\left\|\partial_{z} U^{h} ; L^{2}\left(\varpi_{\sharp}^{h}\right)\right\|^{2} \\
& \quad \leq\left(1+h^{-1} \Lambda^{h}\right)\left\|U^{h}(\cdot, 0) ; L^{2}\left(\omega^{0}\right)\right\|^{2} \leq C .
\end{aligned}
$$

Thus, observing that the $H^{1}\left(\varpi_{\sharp}^{1}\right)$-norm of $\mathbf{U}^{h}$ is uniformly bounded, we again find a subsequence of $\left\{h_{j}\right\}_{j \in \mathbb{N}}$ such that

$$
\begin{aligned}
& \mathbf{U}^{h} \rightarrow \widehat{v} \quad \text { weakly in } H^{1}\left(\varpi_{\sharp}^{1}\right), \\
& \mathbf{U}^{h}(\cdot, 0) \rightarrow \widehat{v} \text { strongly in } L^{2}\left(\omega_{\sharp}^{0}\right),
\end{aligned}
$$

where $\widehat{v} \in H^{1}\left(\varpi_{\sharp}^{0}\right)$ is a function independent of $\zeta$ (recall the factor $h^{-1}$ of the norm $\left\|\partial_{\zeta} \mathbf{U}^{h} ; L^{2}\left(\varpi_{\sharp}^{1}\right)\right\|$ in $\left.(53)\right)$. Finally,

$$
I_{\mathrm{le}}^{h}(\psi) \rightarrow \int_{\omega_{\sharp}^{0}} H(y) \nabla_{y} \widehat{v}(y) \cdot \nabla_{y} \psi(y) d y-\widehat{\mu} \int_{\omega_{\sharp}^{0}} H(y) \widehat{v}(y) \cdot \psi(y) d y
$$

and, moreover, from the normalization of $U^{h}$ and the strong convergence in (51) and (54) we derive the equality

$$
\left\|\widehat{v} ; L^{2}\left(\omega_{\sharp}^{0}\right)\right\|^{2}+\left|\widehat{v}_{\theta}\right|^{2} \operatorname{mes}_{2}(\theta)=1 .
$$


Formulas (49) and (52) demonstrate that the limits $\widehat{\mu}$ in (48) and $\widehat{v}$ in (54) satisfy the integral identity (44), thus, in view of (56), $\widehat{v} \in \mathcal{H}(\eta)$ is a non- trivial eigenfunction of the limit problem (44), corresponding to its eigenvalue $\widehat{\mu}$.

Lemma 1 The limits $\widehat{\mu}$ in (48) and $\widehat{v}$ in (54) compose an eigenpair of the limit problem (44), or, (25)-(28), (42).

The Proof of Lemma 1 is completed only in Remark 2 in Sect. 3.4, but on the other hand, it is needed only at the end of the Proof of Theorem 1.

\subsection{Operator formulation of the model problem in the periodicity cell}

The next assertion is known as the "lemma on almost eigenvalues and eigenvectors" [29] and it is a consequence of the spectral decomposition of the resolvent, see, e.g. [5, Ch. 6].

Lemma 2 Let the function $\mathcal{U}^{h} \in \mathcal{H}_{\eta}^{h}$ and number $k^{h} \in \mathbb{R}_{+}$be such that

$$
\left\|\mathcal{U}^{h} ; \mathcal{H}_{\eta}^{h}\right\|=1 \text { and }\left\|\mathcal{K}_{\eta}^{h} \mathcal{U}^{h}-k^{h} \mathcal{U}^{h} ; \mathcal{H}_{\eta}^{h}\right\|=: \delta^{h} \in\left(0, k^{h}\right)
$$

Then, there exists an eigenvalue $\kappa_{m}^{h}$ (with some index $m$ ) of the operator $\mathcal{K}_{\eta}^{h}$ such that

$$
\left|k^{h}-\kappa_{m}^{h}\right| \leq \delta^{h}
$$

Furthermore, for any $\delta_{*}^{h} \in\left(\delta^{h}, k^{h}\right)$ one can find a column of coefficients $b^{h}=$ $\left(b_{M^{h}}^{h}, \ldots, b_{M^{h}+X^{h}-1}^{h}\right)$ satisfying

$$
\left\|\mathcal{U}^{h}-\sum_{p=M^{h}}^{M^{h}+X^{h}-1} b_{p}^{h} u_{p}^{h} ; \mathcal{H}_{\eta}^{h}\right\| \leq \frac{\delta^{h}}{\delta_{*}^{h}}, \quad \sum_{p=M^{h}}^{M^{h}+X^{h}-1}\left|b_{p}^{h}\right|^{2}=1,
$$

where the sequence $\kappa_{M^{h}}^{h}, \ldots, \kappa_{M^{h}+X^{h}-1}^{h}$ consists of all eigenvalues of $\mathcal{K}_{\eta}^{h}$ contained in the closed interval $\left[k^{h}-\delta_{*}^{h}, k^{h}+\delta_{*}^{h}\right]$ and $u_{M^{h}}^{h}, \ldots, u_{M^{h}+X^{h}-1}^{h}$ are the corresponding eigenvectors, which are orthonormalized in $\mathcal{H}_{\eta}^{h}$.

\subsection{Global approximation and estimation of the discrepancies}

To glue the outer and inner expansion constructed in Sect. 2 we employ a trick, which uses cut-off functions with overlapping supports, see [16, Ch. 2] and [19] and Fig. 2b). Consequently, we define the following smooth functions

$$
\begin{aligned}
& X^{h}(x)=1 \text { for } x \in \varpi_{\sharp}^{h}, n<-2 h, \\
& \quad X^{h}(x)=0 \text { for } x \in \Theta \text { or } x \in \varpi_{\sharp}^{h}, n>-h ; \\
& \chi(x)=1 \text { for } x \in \varpi_{\sharp}^{h} \cap \mathcal{V}, n>-r_{0} \text { or } x \in \Theta \cap \mathcal{V}, r<r_{0},
\end{aligned}
$$




$$
\begin{aligned}
& \chi(x)=0 \text { for } x \in \varpi_{\sharp}^{h}, n<-2 r_{0} \text { or } x \in \Theta, r>2 r_{0} ; \\
& \mathcal{X}^{h}(x)=1 \text { for } x \in \Theta, r>2 H_{\theta} h, \\
& \mathcal{X}^{h}(x)=0 \text { for } x \in \varpi_{\sharp}^{h} \text { or } x \in \Theta, r<H_{\theta} h .
\end{aligned}
$$

Here, $r_{0}$ is a positive number so small that $\left\{x \in \varpi^{h}: r<2 r_{0}\right\} \subset \mathcal{V}$ and $H_{\theta}=$ $\max _{y \in \partial \theta} H(y)$ and will be finally fixed only in Lemma 5 .

If $\{\mu(\eta), v(\eta ; \cdot)\}$ is an eigenpair of the limit problem (25)-(28), (42), formulas (16) and (21) suggest to take

$$
k^{h}(\eta)=h^{-1}(\mu(\eta)+1)^{-1}
$$

as the "almost eigenvalue" of the operator $\mathcal{K}_{\eta}^{h}$. In the following we will omit $\eta$ from the notation.

We take the following composite function as an "almost eigenvector":

$$
\begin{aligned}
\mathcal{W}^{h}= & X^{h}(x)\left(v^{0}(y)+h^{2} v^{\prime}\left(y, h^{-1} z\right)\right) \\
& +\chi(x)\left(a^{0}+h H(y) \partial_{n} v^{0}(s, 0) W\left(h^{-1} H(y)^{-1} n, h^{-1} H(y)^{-1} z\right)-h a_{h}^{1}(s)\right) \\
& -X^{h}(x) \chi(x)\left(v^{0}(s, 0)+n \partial_{n} v^{0}(s, 0)\right), \quad x \in \varpi_{\sharp}^{h} ; \\
\mathcal{W}^{h}= & \mathcal{X}^{h}(x)\left(V^{0}+h V^{\prime}(x)\right) \\
& +\chi(x)\left(a^{0}+h H(s) \partial_{n} v^{0}(s, 0) W\left(h^{-1} H(s)^{-1} n, h^{-1} H(s)^{-1} z\right)-h a_{h}^{1}(s)\right) \\
& -\mathcal{X}^{h}(x) \chi(x)\left(a^{0}+h H(s) \partial_{n} v^{0}(s, 0) \frac{2}{\pi} \ln \frac{r}{h H(s)}\right), \quad x \in \Theta .
\end{aligned}
$$

Let us explain the complicated structure of this global asymptotic approximation of the eigenfunction $v(\eta, \cdot)$.

- In both expressions (64) and (65) the first two lines on the right-hand sides contain terms which have been matched in Sect. 2, but subtracting the terms on the third lines (with factors $X^{h} \chi$ and $\mathcal{X}^{h} h$, respectively), compensates such a duplication.

- The function $v^{\prime}$ is found as a solution of the problem (24) and it is made unique by imposing the orthogonality condition

$$
\int_{-H(y)}^{0} v^{\prime}(y, \zeta) d \zeta=0, \quad y \in \omega_{\sharp}^{0} .
$$

- The arguments of the inner term (37) are different in (64) and (65), but nevertheless the function $\mathcal{W}^{h}$ belongs to $H^{1}\left(\varpi^{h}\right)$ because $H(y)$ becomes $H(s)$ on the common boundary surface (30) of $\varpi_{\sharp}^{h}$ and $\Theta$. 
- The constant $a^{0}=V^{0}$ is taken from (33) and (40). The behavior of $V^{\prime}$ near the edge $\partial \theta$ is described by the formula

$$
V^{\prime}(x)=\frac{2}{\pi} H(s) \partial_{n} v(s, 0) \ln r+V_{0}^{\prime}(s)+\tilde{V}^{\prime}(x), \quad x \in \Theta \cap \mathcal{V},
$$

cf. (41). Here, $V_{0}^{\prime} \in C^{\infty}(\partial \theta)$ and

$$
\mid \partial_{z}^{k} \partial_{n}^{\ell} \partial_{s}^{m} \widetilde{V}^{\prime}(x) A \leq c_{k, \ell, m} r^{1-k-\ell}(1+|\ln r|), \quad x \in \Theta \cap \mathcal{V}
$$

the orthogonality condition

$$
\int_{\Theta} V^{\prime}(x) d x=0
$$

makes both $V^{\prime}$ and $V_{0}^{\prime}$ uniquely defined.

- The function $a_{h}^{1}$ in (65) is determined by

$$
a_{h}^{1}(s)=V_{0}^{\prime}(s)-H(s) \partial_{n} v(s, 0) \frac{2}{\pi} \ln (h H(s)) .
$$

Recalling our definitions (64) and (60), (61), we see that the function $\mathcal{W}^{h}$ coincides with $v(y)+h^{2} v^{\prime}(y, \zeta)$ near that ends $\left\{x \in \partial \varpi^{h}: y_{1}= \pm 1 / 2\right\}$ of the cell (7), and therefore, satisfies the quasi-periodicity condition (11) because (27), (28) hold for $v$ as well as $v^{\prime}$. Thus, the function $\mathcal{W}^{h}$ lies in $H_{\eta}^{1}\left(\varpi^{h}\right)$ and we can normalize it as

$$
\mathcal{U}^{h}=\left\|\mathcal{W}^{h} ; \mathcal{H}_{\eta}^{h}\right\|^{-1} \mathcal{W}^{h}
$$

Let us evaluate the quantity $\delta^{h},(57)$, defined by a couple $\left\{k^{h}, \mathcal{U}^{h}\right\}$, using (14), (15) and (63)

$$
\begin{aligned}
\delta^{h}= & \left\|\mathcal{K}_{\eta}^{h} \mathcal{U}^{h}-k^{h} \mathcal{U}^{h} ; H_{\eta}^{h}\right\|=\sup \left|\left\langle\mathcal{K}_{\eta}^{h} \mathcal{U}^{h}-k^{h} \mathcal{U}^{h}, \mathcal{V}^{h}\right\rangle_{h}\right| \\
= & k^{h}\left\|\mathcal{W}^{h} ; \mathcal{H}_{\eta}^{h}\right\|^{-1} \sup \mid(h+h \mu)\left(\mathcal{W}^{h}, \mathcal{V}^{h}\right)_{\omega^{0}} \\
& -\left(\nabla \mathcal{W}^{h}, \nabla \mathcal{V}^{h}\right)_{\varpi^{h}}-h\left(\mathcal{W}^{h}, \mathcal{V}^{h}\right)_{\omega^{0}} \mid \\
= & h^{-1}(1+\mu)^{-1}\left\|\mathcal{W}^{h} ; \mathcal{H}_{\eta}^{h}\right\|^{-1} \sup \mid\left(\nabla \mathcal{W}^{h}, \nabla \mathcal{V}^{h}\right)_{\varpi^{h}} \\
& -h \mu\left(\mathcal{W}^{h}, \mathcal{V}^{h}\right)_{\omega^{0}} \mid,
\end{aligned}
$$

where the supremum is computed over the unit ball of $\mathcal{H}_{\eta}^{h}$ so that we have

$$
\left\|\mathcal{V}^{h} ; \mathcal{H}_{\eta}^{h}\right\| \leq 1
$$

Our aim is to show that $\delta^{h} \leq c(1+|\ln h|)$. This will follow in Corollary 1 from Lemmas 3 and 5. 
Lemma 3 We have

$$
\left|\left(\nabla \mathcal{W}^{h}, \nabla \mathcal{V}^{h}\right)_{\varpi^{h}}-h \mu\left(\mathcal{W}^{h}, \mathcal{V}^{h}\right)_{\omega^{0}}\right| \leq c h^{3 / 2}(1+|\ln h|)
$$

for all $\mathcal{V}^{h}$ belonging to the unit ball of $\mathcal{H}_{\eta}^{h}$.

Proof To evaluate the right-hand side of (70), we write

$$
\begin{aligned}
& \left(\nabla \mathcal{W}^{h}, \nabla \mathcal{V}^{h}\right)_{\varpi}-h \mu\left(\mathcal{W}^{h}, \mathcal{V}^{h}\right)_{\omega^{0}} \\
& =I_{\varpi}\left(\mathcal{V}^{h}\right)+I_{\Theta}\left(\mathcal{V}^{h}\right)+I_{\Xi}\left(\mathcal{V}^{h}\right)+I_{a}\left(\mathcal{V}^{h}\right),
\end{aligned}
$$

where the terms will be defined in the following. First of all, we set

$$
\begin{aligned}
I_{\varpi}\left(\mathcal{V}^{h}\right)= & \left(\nabla\left(v^{0}+h^{2} v^{\prime}\right), \nabla\left(X^{h} \mathcal{V}^{h}\right)\right)_{\varpi_{\sharp}^{h}}-h \mu\left(v^{0}+h^{2} v^{\prime}, X^{h} \mathcal{V}^{h}\right)_{\omega_{\sharp}^{h}} \\
& +\left(\left(v^{0}+h^{2} v^{\prime}\right) \nabla X^{h}, \nabla \mathcal{V}^{h}\right)_{\varpi_{\sharp}^{h}}-\left(\nabla\left(v^{0}+h^{2} v^{\prime}\right), \mathcal{V}^{h} \nabla X^{h}\right)_{\varpi_{\sharp}^{h}} \\
=: & I_{\varpi}^{1}\left(\mathcal{V}^{h}\right)+I_{\varpi}^{2}\left(\mathcal{V}^{h}\right)+I_{\varpi}^{3}\left(\mathcal{V}^{h}\right)+I_{\varpi}^{4}\left(\mathcal{V}^{h}\right) .
\end{aligned}
$$

Here, we commuted the gradient operator $\nabla$ with the cut-off function $X^{h}$. Observing that, by (60), $X^{h} \mathcal{V}^{h}=0$ on $v^{h}$ and integrating by parts yield

$$
\begin{aligned}
& I_{\varpi}^{1}\left(\mathcal{V}^{h}\right)+I_{\varpi}^{2}\left(\mathcal{V}^{h}\right) \\
& \left.=-\left(\Delta\left(v^{0}+h^{2} v^{\prime}\right), X^{h} \mathcal{V}^{h}\right)_{\varpi_{\sharp}^{h}}+\left(\partial_{z}-h \mu\right)\left(v^{0}+h^{2} v^{\prime}\right), X^{h} \mathcal{V}^{h}\right)_{\omega_{\sharp}^{0}} \\
& \quad+\left(\partial_{\nu}\left(v^{0}+h^{2} v^{\prime}\right), X^{h} \mathcal{V}^{h}\right)_{\varpi_{\sharp}^{h}} .
\end{aligned}
$$

The consideration in Sect. 2.1 gives the relations

$$
\begin{aligned}
& \Delta\left(v^{0}+h^{2} v^{\prime}\right)=h^{2} \Delta_{y} v^{\prime}, \quad\left(\partial_{z}-h \mu\right)\left(v^{0}+h^{2} v^{\prime}\right)=-h^{3} \mu v^{\prime}, \\
& \left(1+h^{2}\left|\nabla_{y} H\right|^{2}\right)^{1 / 2}\left(\partial_{\nu} v^{0}+h^{2} v^{\prime}\right)=-h^{3} \nabla_{y} H \cdot \nabla_{y} v^{\prime} .
\end{aligned}
$$

Hence, using the evident estimate

$$
\begin{aligned}
& \left\|\mathcal{V}^{h} ; L^{2}\left(\varpi_{\sharp}^{h}\right)\right\|^{2}+h\left\|\mathcal{V}^{h} ; L^{2}\left(\omega_{\sharp}^{h}\right)\right\|^{2} \\
& \quad \leq c\left(h^{2}\left\|\partial_{z} \mathcal{V}^{h} ; L^{2}\left(\varpi_{\sharp}^{h}\right)\right\|^{2}+h\left\|\mathcal{V}^{h} ; L^{2}\left(\omega_{\sharp}^{0}\right)\right\|^{2}\right) \leq c,
\end{aligned}
$$

see (71), gives us

$$
\begin{aligned}
& \left|I_{\varpi}^{1}\left(\mathcal{V}^{h}\right)+I_{\varpi}^{2}(\mathcal{V})\right| \\
& \leq \quad c\left(h^{2}\left(\operatorname{mes}_{3}\left(\varpi_{\sharp}^{h}\right)\right)^{1 / 2}\left\|\mathcal{V}^{h} ; L^{2}\left(\varpi_{\sharp}^{h}\right)\right\|+h^{3}\left(\operatorname{mes}_{2}\left(\omega_{\sharp}^{0}\right)\right)^{1 / 2}\left\|\mathcal{V}^{h} ; L^{2}\left(\omega_{\sharp}^{0}\right)\right\|\right. \\
& \left.\quad+h^{2}\left(\operatorname{mes}_{2}\left(\omega_{\sharp}^{h}\right)\right)^{1 / 2}\left\|\mathcal{V}^{h} ; L^{2}\left(\omega_{\sharp}^{h}\right)\right\|\right) \leq c\left(h^{2} h^{1 / 2}+h^{3} h^{-1 / 2}+h^{3} h^{-1 / 2}\right) \\
& =3 c h^{5 / 2} .
\end{aligned}
$$


The remaining terms $I_{\varpi}^{3}\left(\mathcal{V}^{h}\right)$ and $I_{\varpi}^{4}\left(\mathcal{V}^{h}\right)$ will be taken into account later.

On the massive domain $\Theta$ we set

$$
\mathcal{V}^{h}(x)=\mathcal{V}_{0}^{h}+\mathcal{V}_{\perp}^{h}(x), \text { where } \int_{\Theta} \mathcal{V}_{\perp}^{h}(x) d x=0,
$$

and using definition (14) together with the Poincaré and trace inequalities, cf. (50), we obtain

$$
h^{1 / 2}\left|\mathcal{V}_{0}^{h}\right|+\left\|\mathcal{V}_{\perp}^{h} ; H^{1}(\Theta)\right\| \leq c\left\|\mathcal{V}^{h} ; \mathcal{H}_{\eta}^{h}\right\| \leq c
$$

Then, we define and estimate

$$
\begin{aligned}
I_{\Theta}\left(\mathcal{V}^{h}\right)= & \left(\nabla\left(\mathcal{X}^{h}\left(V^{0}+h V^{\prime}\right)\right), \nabla \mathcal{V}^{h}\right)_{\Theta}-h \mu\left(\mathcal{X}^{h}\left(V^{0}+h V^{\prime}\right), \mathcal{V}^{h}\right)_{\theta} \\
= & -\left(\Delta\left(V^{0}+h V^{\prime}\right), \mathcal{X}^{h} \mathcal{V}_{\perp}^{h}\right)_{\Theta}+\left(\partial_{\nu}\left(V^{0}+h V^{\prime}\right), \mathcal{X}^{h} \mathcal{V}_{\perp}^{h}\right)_{\partial \Theta \backslash \theta} \\
& \left.+\left(-\partial_{\nu}-h \mu\right)\left(V^{0}+h V^{\prime}\right), \mathcal{X}^{h} \mathcal{V}_{\perp}^{h}\right)_{\theta}-h \mu\left(\mathcal{X}^{h}\left(V^{0}+h V^{\prime}\right), \mathcal{V}_{0}^{h}\right)_{\theta} \\
& +\left(\left(V^{0}+h V^{\prime}\right) \nabla \mathcal{X}^{h}, \nabla \mathcal{V}_{\perp}^{h}\right)_{\Theta}-\left(\nabla\left(V^{0}+h V^{\prime}\right), \mathcal{V}_{\perp}^{h} \nabla \mathcal{X}^{h}\right)_{\Theta} \\
= & : \sum_{j=1}^{6} I_{\Theta}^{j}\left(\mathcal{V}^{h}\right) .
\end{aligned}
$$

Recall that $V^{0}$ is constant and that, by Sect. 2,

$$
\begin{gathered}
\Delta V^{\prime}=0 \text { in } \Theta, \quad \partial_{\nu} V^{\prime}=0 \text { in } \partial \Theta \backslash \theta, \quad\left(\partial_{z}-h \mu\right)\left(V^{0}+h V^{\prime}\right)=-h^{2} \mu V^{\prime} \text { in } \theta, \\
\left|V^{\prime}(y, 0)\right| \leq c(1+|\ln r|) \leq c(1+|\ln h|) \text { for } y \in \theta \cap \operatorname{supp} \mathcal{X}^{h},
\end{gathered}
$$

hence, we have

$$
\begin{aligned}
& I_{\Theta}^{1}\left(\mathcal{V}^{h}\right)=0, \quad I_{\Theta}^{2}\left(\mathcal{V}^{h}\right)=0, \\
& \quad\left|I_{\Theta}^{3}\left(\mathcal{V}^{h}\right)\right| \leq c h^{2}(1+|\ln h|)\left\|\mathcal{V}_{\perp}^{h} ; L^{2}(\theta)\right\| \leq c h^{2}(1+|\ln h|) .
\end{aligned}
$$

The last two terms will be treated in the next paragraph, while the estimate (76) yields

$$
\begin{aligned}
& \left|I_{\Theta}^{4}\left(\mathcal{V}^{h}\right)-h \mu V^{0} \overline{\mathcal{V}_{0}^{h}} \operatorname{mes}_{2}(\theta)\right| \\
& \quad=h \mu\left|\left(\left(1-\mathcal{X}^{h}\right) V^{0}, \mathcal{V}^{h}\right)_{\theta}+h\left(\mathcal{X}^{h} V^{\prime}, \mathcal{V}^{h}\right)_{\theta}\right| \\
& \quad \leq \operatorname{ch}\left(\operatorname{mes}_{2}\left(\theta_{\mathcal{X}}^{h}\right)\left|V^{0}\right|+h\left\|V^{\prime} ; L^{2}(\theta)\right\|\right)\left\|\mathcal{V}^{h} ; \mathcal{H}_{\eta}^{h}\right\| \\
& \quad \leq \operatorname{ch}(h+h) h^{-1 / 2}\left\|\mathcal{V}^{h} ; \mathcal{H}_{\eta}^{h}\right\| \leq 2 c h^{3 / 2},
\end{aligned}
$$

where, according to (62),

$$
\theta_{\mathcal{X}}^{h}=\left\{y \in \theta: \mathcal{X}^{h}(y, 0) \neq 1\right\} \subset\left\{y \in \theta: r<2 H_{\theta} h\right\} .
$$


The last couples of terms in (74) and (77) will be compensated by parts of the expression

$$
\begin{aligned}
& \left.I_{a}\left(\mathcal{V}^{h}\right)=\left(\nabla\left(X^{h} \chi\left(v_{\theta}^{0}+n \partial_{z} v_{\theta}^{0}\right)\right), \nabla v^{h}\right)_{\varpi_{\sharp}^{h}}-h \mu\left(X^{h} \chi\left(v_{\theta}^{0}+n \partial_{n} v_{\theta}^{0}\right)\right), \mathcal{V}^{h}\right)_{\omega_{\sharp}^{0}} \\
& \quad+\left(\nabla\left(\mathcal{X}^{h} \chi\left(a^{0}+h A_{h}-h a^{1}\right), \nabla \mathcal{V}^{h}\right)_{\Theta}\right. \\
& \quad-h \mu\left(\mathcal{X}^{h} \chi\left(a^{0}+h A_{h}-h a^{1}\right), \mathcal{V}^{h}\right)_{\theta},
\end{aligned}
$$

where $\partial_{n} v_{\theta}(s)=\partial_{n} v^{0}(s, 0)$ and $A_{h}(s, r)=H(s) \partial_{n} v_{\theta}^{0}(s) \frac{2}{\pi} \ln \frac{r}{h H(s)}$. Taking into account the position of the supports in (60)-(62) shows that

$$
\nabla\left(X^{h} \chi\right)=\nabla X^{h}+\nabla \chi, \quad \nabla\left(\mathcal{X}^{h} \chi\right)=\nabla \mathcal{X}^{h}+\nabla \chi
$$

and we rewrite (78) as follows:

$$
\begin{aligned}
I_{a}\left(\mathcal{V}^{h}\right) & \\
= & -\left(\nabla\left(v_{\theta}^{0}+n \partial_{n} v_{\theta}^{0}\right), \nabla\left(X^{h} \chi \mathcal{V}^{h}\right)\right)_{\varpi_{\sharp}^{h}}+h \mu\left(v_{\theta}^{0}+n \partial_{n} v_{\theta}^{0}, X^{h} \chi \mathcal{V}^{h}\right)_{\omega_{\sharp}^{h}} \\
& -\left(\left(v_{\theta}^{0}+n \partial_{n} v_{\theta}^{0}\right) \nabla X^{h}, \nabla \mathcal{V}^{h}\right)_{\varpi_{\sharp}^{h}}+\left(\nabla\left(v_{\theta}^{0}+n \partial_{n} v_{\theta}^{0}\right), \mathcal{V}^{h} \nabla X^{h}\right)_{\varpi_{\sharp}^{h}} \\
& -\left(\left(v_{\theta}^{0}+n \partial_{n} v_{\theta}^{0}\right) \nabla \chi, \nabla \mathcal{V}^{h}\right)_{\varpi_{\sharp}^{h}}+\left(\nabla\left(v_{\theta}^{0}+n \partial_{n} v_{\theta}^{0}\right), \mathcal{V}^{h} \nabla \chi\right)_{\varpi_{\sharp}^{h}} \\
& -\left(\nabla\left(a^{0}+h A_{h}-h a_{h}^{1}\right), \nabla\left(\mathcal{X}^{h} \chi \mathcal{V}_{\perp}^{h}\right)\right)_{\Theta}+h \mu\left(a^{0}+h A_{h}-h a_{h}^{1}, \mathcal{X}^{h} \chi \mathcal{V}^{h}\right)_{\theta} \\
& -\left(\left(a^{0}+h A_{h}-h a_{h}^{1}\right) \nabla \mathcal{X}^{h}, \nabla \mathcal{V}_{\perp}^{h}\right)_{\Theta}+\left(\nabla\left(a^{0}+h A_{h}-h a_{h}^{1}\right), \mathcal{V}^{h} \nabla \mathcal{X}_{\perp}^{h}\right)_{\Theta} \\
& -\left(\nabla\left(a^{0}+h A_{h}-h a_{h}^{1}\right) \nabla \chi, \nabla \mathcal{V}_{\perp}^{h}\right)_{\Theta}+\left(\nabla\left(a^{0}+h A_{h}-h a_{h}^{1}\right), \mathcal{V}^{h} \nabla \chi\right)_{\Theta} \\
= & : \sum_{j=1}^{12} I_{a}^{j}\left(\mathcal{V}^{h}\right) .
\end{aligned}
$$

In view of (60) and (62) we have

$$
\begin{gathered}
n=O(h) \text { in } T^{h}=\operatorname{supp}\left|\nabla X^{h}\right|, \quad r=O(h) \text { in } \mathcal{T}^{h}=\operatorname{supp}\left|\nabla \mathcal{X}^{h}\right|, \\
\left|\nabla X^{h}(x)\right|+\left|\nabla \mathcal{X}^{h}(x)\right| \leq c h^{-1}, \quad \operatorname{mes}_{3} T^{h}+\operatorname{mes}_{3} \mathcal{T}^{h} \leq c h^{2} .
\end{gathered}
$$

Hence, using the relations

$$
\begin{aligned}
& \left|v^{0}(y)-v_{\theta}^{0}(s)-n \partial_{n} v_{\theta}^{0}(s)\right| \leq c(\operatorname{dist}(y, \partial \theta))^{2}, \quad\left|v^{\prime}(y, \zeta)\right| \leq c, \\
& V^{0}=a^{0}, \quad\left|V^{0}(x)+h V^{\prime}(x)-a^{0}-h A_{h}(s, r)+h a_{h}^{1}(s)\right| \leq c \operatorname{dist}(x, \partial \theta),
\end{aligned}
$$

we conclude that

$$
\begin{gathered}
\left|I_{\varpi}^{3}\left(\mathcal{V}^{h}\right)+I_{a}^{3}\left(\mathcal{V}^{h}\right)\right| \leq c h^{-1}\left(\operatorname{mes}_{3} T^{h}\right)^{1 / 2} h^{2}\left\|\nabla \mathcal{V}^{h} ; L^{2}\left(T^{h}\right)\right\| \leq c h^{2}, \\
\left|I_{\Theta}^{5}\left(\mathcal{V}^{h}\right)+I_{a}^{9}\left(\mathcal{V}^{h}\right)\right| \leq c h^{-1}\left(\operatorname{mes}_{3} \mathcal{T}^{h}\right)^{1 / 2} h^{2}\left\|\nabla \mathcal{V}^{h} ; L^{2}\left(\mathcal{T}^{h}\right)\right\| \leq c h^{2} .
\end{gathered}
$$


The estimates

$$
\begin{aligned}
& \left|I_{\varpi}^{4}\left(\mathcal{V}^{h}\right)+I_{a}^{4}\left(\mathcal{V}^{h}\right)\right| \leq c h^{-1}\left(\operatorname{mes}_{3} T^{h}\right)^{1 / 2} h\left\|\mathcal{V}^{h} ; L^{2}\left(T^{h}\right)\right\| \leq c h^{3 / 2} \\
& \left|I_{\Theta}^{6}\left(\mathcal{V}^{h}\right)+I_{a}^{10}\left(\mathcal{V}^{h}\right)\right| \leq c h^{-1}\left(\operatorname{mes}_{3} \mathcal{T}^{h}\right)^{1 / 2} h\left\|\mathcal{V}_{\perp}^{h} ; L^{2}\left(\mathcal{T}^{h}\right)\right\| \leq \\
\leq & c h^{3 / 2}(1+|\ln h|)
\end{aligned}
$$

are obtained in the same way, but with the help of the next lemma. The remaining terms in (79) will be considered later, but notice that until now all terms of (74) and (77) have been taken into account.

Lemma 4 The following inequalities hold true:

$$
\begin{aligned}
& \left\|\mathcal{V}^{h} ; L^{2}\left(T^{h}\right)\right\| \leq c h^{1 / 2}\left\|\mathcal{V}^{h} ; \mathcal{H}_{\eta}^{h}\right\| \\
& \left\|\mathcal{V}_{\perp}^{h} ; L^{2}\left(\mathcal{T}^{h}\right)\right\| \leq c h^{1 / 2}(1+|\ln h|)\left\|\mathcal{V}_{\perp}^{h} ; H^{1}(\Theta)\right\|
\end{aligned}
$$

Proof of Lemma 4 First of all, repeating the calculation (53) with an evident modification yields

$$
\left\|\mathcal{V}^{h} ; L^{2}\left(\varpi_{\sharp}^{h}\right)\right\|^{2} \leq c\left(h\left\|\mathcal{V}^{h} ; L^{2}\left(\omega_{\sharp}^{0}\right)\right\|^{2}+h^{2}\left\|\partial_{z} \mathcal{V}^{h} ; L^{2}\left(\varpi_{\sharp}^{h}\right)\right\|^{2} \leq\left\|\mathcal{V}^{h} ; \mathcal{H}_{\eta}^{h}\right\|^{2} .\right.
$$

Then, we write the Newton-Leibnitz formula in the form

$$
\mathcal{V}^{h}(s, n, z)=\int_{n_{0}}^{n} \frac{\partial}{\partial t}\left(\chi_{\varpi}(t) \mathcal{V}^{h}(s, t, z)\right) d t
$$

where $n_{0}>0$ is fixed such that $\left\{x \in \varpi_{\sharp}^{h}: n>-n_{0}\right\} \subset v$ and $\chi_{\varpi}$ is a smooth cut-off function,

$$
\chi_{\varpi}(n)=1 \text { for } n>-n_{0} / 3, \quad \chi_{\varpi}(n)=0 \text { for } n<-2 n_{0} / 3 .
$$

Integrating (83) over $(-2 h, 0) \ni n, \partial \theta \ni s$ and $(-h H(y), 0) \ni z$, in other words over $T^{h} \ni x$, leads to the inequality

$$
\int_{T^{h}}\left|\mathcal{V}^{h}(x)\right|^{2} d x \leq \operatorname{ch} \int_{\varpi_{\sharp}^{h}}\left(\left|\nabla \mathcal{V}^{h}(x)\right|^{2}+\left|\mathcal{V}^{h}(x)\right|^{2}\right) d x,
$$

which is nothing but the first inequality in (81).

The second inequality in (81) follows from the estimate

$$
\left\|\mathcal{V}_{\perp}^{h} ; H^{1}(\Theta)\right\| \leq c\left\|\mathcal{V}^{h} ; \mathcal{H}_{\eta}^{h}\right\|
$$


cf.(50), and the one-dimensional Hardy inequality with logarithm

$$
\int_{0}^{r_{0}}\left|\ln \frac{r}{r_{0}}\right|^{-2}|\mathbf{V}(r)|^{2} \frac{d r}{r} \leq 4 \int_{0}^{r_{0}}\left|\frac{d \mathbf{V}}{d r}\right|^{2} r d r
$$

and the fact that $r^{-2}|\ln r|^{-2} \geq c h^{-2}(1+|\ln h|)^{-2}$ for $x \in \mathcal{T}^{h}$. $\otimes$

We complete the Proof of Lemma 3. Let us write the term $I_{\Xi}\left(\mathcal{V}^{h}\right)$ as follows:

$$
\begin{aligned}
I_{\Xi}\left(\mathcal{V}^{h}\right)= & \left(\nabla \mathbf{W}_{\varpi}, \nabla\left(\chi \mathcal{V}^{h}\right)\right)_{\varpi_{\sharp}^{h}}-h \mu\left(\mathbf{W}_{\varpi}, \chi \mathcal{V}^{h}\right)_{\omega_{\sharp}^{0}} \\
& +\left(\nabla W_{\Theta}, \nabla\left(\chi \mathcal{V}^{h}\right)\right)_{\Theta}-h \mu\left(\mathbf{W}_{\Theta}, \chi \mathcal{V}^{h}\right)_{\theta} \\
& +\left(\mathbf{W}_{\varpi} \nabla \chi, \nabla \mathcal{V}^{h}\right)_{\varpi_{\sharp}^{h}}-\left(\nabla \mathbf{W}_{\varpi}, \mathcal{V}^{h} \nabla \chi\right)_{\varpi_{\sharp}^{h}} \\
& +\left(\mathbf{W}_{\varpi} \nabla \chi, \nabla \mathcal{V}^{h}\right)_{\Theta}-\left(\nabla \mathbf{W}_{\Theta}, \mathcal{V}^{h} \nabla \chi\right)_{\Theta}=\sum_{k=1}^{8} I_{\Xi}\left(\mathcal{V}^{h}\right),
\end{aligned}
$$

where $\mathbf{W}_{\varpi}$ and $\mathbf{W}_{\Theta}$ are the multipliers of $\chi$ in the middle of the right-hand sides in (64) and (65), respectively; recall that the arguments of $W$ in these sums differ from each other.

We treat the sums (84) and (79) together and start with the simplest terms. According to (37) and (34), the modulus of the difference

$$
\begin{aligned}
& h H(y) \partial_{n} v^{0}(s, 0) W\left(h^{-1} H(y)^{-1} n, h^{-1} H(y)^{-1} z\right)-n \partial_{n} v^{0}(s, 0) \\
& \quad=h H(y) \partial_{n} v^{0}(s, 0) c_{\Xi}+O\left(e^{-\pi|n| /(h H(y)}\right)
\end{aligned}
$$

is bounded by $c h$, we obtain

$$
\left|I_{\Xi}^{5}\left(\mathcal{V}^{h}\right)+I_{a}^{5}\left(\mathcal{V}^{h}\right)\right| \leq \operatorname{ch}\left(\operatorname{mes}_{3} \varpi_{\sharp}^{h}\right)^{1 / 2}\left\|\mathcal{V}^{h} ; L^{2}\left(\varpi_{\sharp}^{h}\right)\right\| \leq c h^{3 / 2} .
$$

In a similar way, by (66) and (67) we observe that the expression

$$
\begin{gathered}
h H(s) \partial_{n} v^{0}(s, 0) W\left(h^{-1} H(s)^{-1} n, h^{-1} H(s)^{-1} z\right) \\
-h a_{h}^{1}(s)-h H(s) v^{0}(s, 0) \frac{2}{\pi} \ln \frac{r}{h H(s)}
\end{gathered}
$$

is $O\left(h^{2} r(1+|\ln r|)\right)$ in $\Theta$ and thus $O\left(h^{2}\right)$ in supp $|\nabla \chi|$. Hence,

$$
\left|I_{\Xi}^{7}\left(\mathcal{V}^{h}\right)+I_{a}^{11}\left(\mathcal{V}^{h}\right)\right| \leq c h^{2}\left\|\mathcal{V}_{\perp}^{h} ; L^{2}(\Theta)\right\| \leq c h^{2} .
$$

As for the pairs $I_{\Xi}^{6}\left(\mathcal{V}^{h}\right), I_{a}^{6}\left(\mathcal{V}^{h}\right)$ and $I_{\Xi}^{8}\left(\mathcal{V}^{h}\right), I_{a}^{12}\left(\mathcal{V}^{h}\right)$, we have to take into account the formula

$$
\nabla=\left((1+n \varkappa(s))^{-1} \partial_{s}, \partial_{n}, \partial_{z}\right)
$$


We observe that none of the three derivatives of (88) affects the above presented boundedness for the expression (85). The same holds concerning the estimate for the bounded of the expression (87), except on the set supp $|\nabla \chi|$, the position of which is however at a positive distance from the edge $\partial \theta$ containing the singularities of the solutions. Thus, we conclude that

$$
\begin{gathered}
\left|I_{\Xi}^{6}\left(\mathcal{V}^{h}\right)+I_{a}^{6}\left(\mathcal{V}^{h}\right)\right| \leq c h^{3 / 2}\left\|\mathcal{V}^{h} ; L^{2}\left(\varpi_{\sharp}^{h}\right)\right\| \leq c h^{3 / 2}, \\
\left|I_{\Xi}^{8}\left(\mathcal{V}^{h}\right)+I_{a}^{12}\left(\mathcal{V}^{h}\right)\right| \leq c h^{2}\left\|\mathcal{V}^{h} ; L^{2}(\Theta)\right\| \leq c h^{3 / 2} .
\end{gathered}
$$

On the first line we also used (82) and on the second one the inequality

$$
\left\|\mathcal{V}^{h} ; L^{2}(\Theta)\right\| \leq c\left(\left\|\nabla \mathcal{V}^{h} ; L^{2}(\Theta)\right\|+\left\|\mathcal{V}^{h} ; L^{2}(\theta)\right\|\right) \leq c h^{-1 / 2}\left\|\mathcal{V}^{h} ; \mathcal{H}_{\eta}^{h}\right\|
$$

coming from the definition of the scalar product in $\mathcal{H}_{\eta}^{h}$.

We are left with the sums

$$
I_{\Xi}^{1}\left(\mathcal{V}^{h}\right)+I_{\Xi}^{3}\left(\mathcal{V}^{h}\right), \quad I_{\Xi}^{2}\left(\mathcal{V}^{h}\right)+I_{\Xi}^{4}\left(\mathcal{V}^{h}\right)
$$

and

$$
I_{a}^{1}\left(\mathcal{V}^{h}\right)+I_{a}^{7}\left(\mathcal{V}^{h}\right), \quad I_{a}^{2}\left(\mathcal{V}^{h}\right)+I_{a}^{8}\left(\mathcal{V}^{h}\right)
$$

in (84) and (79), respectively. The two-dimensional integrals included into the second sums in (90) and (91) are treated using the information we already have on the expressions (85) and (86), which gives us

$$
\begin{gathered}
\left|I_{\Xi}^{2}\left(\mathcal{V}^{h}\right)+I_{a}^{2}\left(\mathcal{V}^{h}\right)\right| \leq c h^{2}\left\|\mathcal{V}^{h} ; L^{2}\left(\omega_{\sharp}^{0}\right)\right\| \leq c h^{3 / 2}, \\
\left|I_{\Xi}^{4}\left(\mathcal{V}^{h}\right)+I_{a}^{8}\left(\mathcal{V}^{h}\right)\right| \leq h^{2}\left\|\mathcal{V}^{h} ; L^{2}(\theta)\right\| \leq c h^{3 / 2} .
\end{gathered}
$$

Here we also applied the inequality $\left\|\mathcal{V}^{h} ; L^{2}\left(\omega^{0}\right)\right\| \leq h^{-1 / 2}\left\|\mathcal{V}^{h} ; \mathcal{H}_{\eta}^{h}\right\|$, which is a consequence of the definition (14).

To conclude with the first sums in (90) and (91), we need to take into account several aspects. To start with, the first component on the gradient operator (88), which acts in the integrals over the sets $\varpi_{\sharp}^{h}$ and $\Theta$, is treated in the same way as in (89); notice that in the bound $c h^{2} r(1+|\ln r|)$ of the modulus of (87), the factor $r(1+|\ln r|)$ is small near the edge $\partial \theta$. Next, the factor $n \varkappa(s)$ in the Jacobian of the differential

$$
d x=(1+n \varkappa(s)) d s d n d z
$$

is also $O(r)$. Together, these compensate the singularities of $\nabla_{\xi} W(\xi)$ of the solution (37) of the Neumann problem (36) at the corner point $P$. 
Finally, recalling that the arguments of the function $W$ are different in (64) and (65), we write

$$
\begin{aligned}
& \frac{\partial}{\partial z}\left(W\left(\frac{n}{h H(y)}, \frac{z}{h H(y)}\right)-W\left(\frac{n}{h H(s)}, \frac{z}{h H(s)}\right)\right)=\frac{1}{h}\left(\frac{1}{H(y)}-\frac{1}{H(s)}\right) \frac{\partial W}{\partial \xi_{2}}(\xi), \\
& \quad \frac{\partial}{\partial n}\left(W\left(\frac{n}{h H(y)}, \frac{z}{h H(y)}\right)-W\left(\frac{n}{h H(s)}, \frac{z}{h H(s)}\right)\right) \\
& =\frac{1}{h}\left(\frac{1}{H(y)}-\frac{1}{H(s)}\right) \frac{\partial W}{\partial \xi_{1}}(\xi)-\frac{n}{h} \frac{\partial_{s} H(y)}{H(y)^{2}} \frac{\partial W}{\partial \xi_{1}}(\xi)-\frac{z}{h} \frac{\partial_{s} H(y)}{H(y)^{2}} \frac{\partial W}{\partial \xi_{2}}(\xi) .
\end{aligned}
$$

Again, in $\varpi_{\sharp}^{h}$ there appears a factor of order $O(n)$, due to $|H(y)-H(s)|=O(n)$, see Sect. 2.2. This and the obvious relation

$$
\int_{0}^{1} n^{2} e^{-2 \delta n / h} d n=O(h), \quad \delta>0,
$$

yield an additional factor $h^{1 / 2}$ in the above estimates, thus compensating the coefficient $h^{-1}$ in (34).

We denote by $\widehat{I}_{\Xi}^{q}\left(\mathcal{V}^{h}\right)$ and $\widehat{I}_{a}^{q}\left(\mathcal{V}^{h}\right)$ the expressions in (90) and (31) after the above mentioned simplifications have been made. We have

$$
\begin{aligned}
& \widehat{I}_{a}^{1}\left(\mathcal{V}^{h}\right)+\widehat{I}_{\Xi}^{3}\left(\mathcal{V}^{h}\right) \\
& =\int_{\partial \theta} \int_{\Xi} \nabla_{\xi}\left(a^{0}+h H(s) \partial_{n} v^{0}(s, 0) W(\xi)-h a_{h}^{1}(s)\right) \cdot \nabla_{\xi}\left(\chi \mathcal{V}^{h}\right) d \xi d s \\
& \widehat{I}_{a}^{2}\left(\mathcal{V}^{h}\right)=-\int_{\partial \theta} \int_{\mathbb{P}} \nabla_{\xi}\left(v^{0}(s, 0)+h \xi_{1} \partial_{n} v^{0}(s, 0)\right) \cdot \nabla_{\xi}\left(\chi X^{h} \mathcal{V}^{h}\right) d \xi d s \\
& \widehat{I}_{a}^{7}\left(\mathcal{V}^{h}\right) \\
& =-\int_{\partial \theta} \int_{\mathbb{K}} \nabla_{\xi}\left(a^{0}+h H(s) \partial_{n} v^{0}(s, 0) \frac{2}{\pi} \ln \frac{r}{h H(s)}\right) \cdot \nabla_{\xi}\left(\chi \mathcal{X}^{h} \mathcal{V}^{h}\right) d \xi d s .(92)
\end{aligned}
$$

These integrals involve several solutions of the Neumann Laplacian in the semi-strip $\mathbb{P}$, the quadrant $\mathbb{K}$ and in their union $\Xi$, see Sect. 2.2, as well as test functions with compact support, which in particular vanish near the end of $\mathbb{P}$ and the corner point of $\mathbb{K}$. As a consequence, the integrals (92) vanish.

This completes the consideration of the terms in (74), (77), (79) and (84). Lemma 3 thus follows by combining the formulas (70)-(74) with the inequalities (75)-(92). $\otimes$

Note that the worst (largest) bound for the left-hand side of (72) appears in (80).

We also need the following estimates for the approximate eigenfunctions.

Lemma 5 Let $\mu_{m}(\eta), m \in \mathbb{N}$, be the eigenvalues (45) and let $v_{m}^{0}(\eta ; \cdot)$ be the corresponding eigenfunctions of the problem (44), orthonormalized as in (46). Then, 
the functions $\mathcal{W}_{m}^{h}$, which are defined in (64), (65) by using $v_{m}^{0}$ and small enough $r_{0}=r_{0}(m)>0$, satisfy for some $\widetilde{h}_{m}>0$ the relation

$$
\left\|\mathcal{W}_{m}^{h}-v_{m}^{0} ; L^{2}\left(\omega^{0}\right)\right\| \leq 2^{-m-2} \forall h \leq \widetilde{h}_{m} .
$$

Consequently, for every $m \geq 2$ and $0<h<\widetilde{h}_{m}$, the sequence $\left(\mathcal{W}_{n}^{h}\right)_{n=1}^{m}$ is linearly independent, and there also holds, for some constants $c_{1}, c_{2}>0$,

$$
c_{1} h^{1 / 2} \leq\left\|\mathcal{W}_{m}^{h} ; \mathcal{H}_{\eta}^{h}\right\| \leq c_{2} h^{1 / 2} \quad \forall h \leq \widetilde{h}_{m}
$$

Proof. Let us consider the defining formulas (64), (65). By standard elliptic regularity results, $[1,2]$, the smoothness of the boundary $\partial \omega_{\sharp}^{0}$, and the definition as the solution of (25)-(28), (42), the functions $v^{0}, \partial_{n} v^{0}, \Delta_{y} v^{0}$ are bounded by a constant independent of $h$ and $\eta$ in the domain $\omega^{0}$ (we again drop the index $m$ from the notation). Consequently, by (24), also $v^{\prime}$ is bounded in the same way.

By (37), we have $W\left(h^{-1} H(s)^{-1} n, h^{-1} H(s)^{-1} z\right) \leq c h^{-1}$, hence,

$$
\left|h H(y) \partial_{n} v^{0}(s, 0) W\left(h^{-1} H(s)^{-1} n, h^{-1} H(s)^{-1} z\right)\right| \leq c^{\prime}
$$

on $\omega^{0}$. The modulus of the function $a_{h}^{1}(s)$ is bounded by $c h|\ln h|$, see (68). Thus, the second and third rows of (64) can be written, by taking $x=(y, 0)$, as $\chi(y) F^{h}(y)$, $y \in \omega_{\sharp}^{0}$, where $F^{h}$ is a function, which is bounded by a constant independent of $h$. Choosing $r_{0}=r_{0}(m)>0$ small enough in the definition of $\chi,(61)$, we get

$$
\left\|\chi F^{h} ; L^{2}\left(\omega_{\sharp}^{0}\right)\right\| \leq 2^{-m-4} .
$$

In the same way, the second and third rows of (65) can be written as $\chi(y) F^{h}(y), y \in \theta$, for a bounded extension of $F^{h}$ into $\theta$. Diminishing the number $r_{0}>0$, if necessary, yields also

$$
\left\|\chi F^{h} ; L^{2}(\theta)\right\| \leq 2^{-m-4} \Rightarrow\left\|\chi F^{h} ; L^{2}\left(\omega^{0}\right)\right\| \leq 2^{-m-3}
$$

for all $h$. Finally, in the subdomain $\widetilde{\omega}^{h}=\left\{y \in \omega^{0}: X^{h}(y)=1\right.$ or $\left.\mathcal{X}^{h}(y)=1\right\}$ we have

$$
\mathcal{W}^{h}=v^{0}+\chi F^{h}+\widetilde{F}^{h},
$$

where $\widetilde{F}^{h}$ equals $h^{2} v^{\prime}(y, 0)$ on $\omega_{\sharp}^{0}$ and $h V^{\prime}$ on $\theta$ so that $\left\|\widetilde{F}^{h} ; L^{2}\left(\omega^{0}\right)\right\| \leq c h$. Since the area of $\omega^{0} \backslash \widetilde{\omega}^{h}$ is $O(h)$ and both functions $\mathcal{W}^{h}$ and $v^{0}$ are bounded, the relation (93) follows from (95) and (96) by choosing $\widetilde{h}_{m}$ small enough.

The claim on the linear independence of the sequence follows from the orthonormality relation (46) (note that the expression on the left-hand side of (46) is nothing but the natural inner product of $L^{2}\left(\omega^{0}\right)$, since the eigenfunctions $v_{m}^{0}$ are constant on $\theta),(93)$ and the well-known result that a small perturbation of a sequence of vectors forming an orthonormal bases is still linearly independent. 
The lower bound in (94) follows from (46), (93) and the definition of the norm of $\mathcal{H}_{\eta}^{h}$ in (14). The upper bound is a matter of a straightforward calculation of the $\mathcal{H}_{\eta}^{h}$-norm of the representation (64)-(65). $\otimes$

We can now conclude with the estimation of $\delta^{h},(70)$ : by (94), the multiplier of the last sup $|\ldots|$ in (70) does not exceed $c h^{-1}\left\|\mathcal{W}^{h} ; \mathcal{H}_{\eta}^{h}\right\|^{-1} \leq C h^{-3 / 2}$. Thus, Lemma 3 yields

Corollary 1 For $0<h<\widetilde{h}$, there holds the bound

$$
\delta^{h} \leq h^{-3 / 2} h^{3 / 2}(1+|\ln h|)=c(1+|\ln h|) .
$$

Here, $\widetilde{h}>0$ means the number $\widetilde{h}_{m}$, found in Lemma 5 for the approximate eigenvector under consideration.

\subsection{Theorem on asymptotics}

Let us state our main asymptotic result on the eigenvalues of the model problem.

Theorem 1 For all $n \in \mathbb{N}$ there exist positive numbers $h_{n}$ and $c_{n}$ such that the entries of the eigenvalue sequences (19) and (45) of the problems (8)-(12) and (44), respectively, are related by

$$
\left|\Lambda_{n}^{h}(\eta)-h \mu_{n}(\eta)\right| \leq c_{n} h^{2}(1+|\ln h|) \quad \forall h \in\left(0, h_{n}\right], \eta \in[-\pi, \pi]
$$

Evidently, the numbers $h_{n}$ and $c_{n}$ can be chosen independently of $\eta$, since the dependence of the eigenvalues $\Lambda_{n}^{h}$ and $\mu_{n}$ on $\eta \in[-\pi, \pi]$ is continuous; cf. [13].

As for the proof, one technical difficulty is caused by the possible higher multiplicities of the eigenvalues of the limit problem. To treat this we need to proceed in several steps and thus start by showing the bound (98) with $\mu_{m}$ in place $\mu_{n}$ for some $m \in \mathbb{N}$, which is for the moment unspecified. In (58) of Lemma 2 and (70), (97) we found an eigenvalue $\kappa_{n}^{h}=\kappa_{n}^{h}(\eta)$ of the model problem such that for an eigenvalue $k_{m}^{h}=k_{m}^{h}(\eta)$ of the limit problem there holds

$$
\left|\kappa_{n}^{h}-k_{m}^{h}\right| \leq c(1+|\ln h|)
$$

We have by (16) and (63),

$$
c(1+|\ln h|) \geq\left|\kappa_{n}^{h}-k_{m}^{h}\right|=\left|\frac{1}{\Lambda_{n}^{h}+h}-\frac{1}{h \mu_{m}+h}\right|
$$

hence,

$$
\begin{gathered}
\left|\Lambda_{n}^{h}-h \mu_{m}\right|=\left|\Lambda_{n}^{h}+h-\left(h \mu_{m}+h\right)\right| \\
\leq c(1+|\ln h|)\left(\Lambda_{n}^{h}+h\right)\left(h \mu_{m}+h\right) .
\end{gathered}
$$


Here, $\mu_{m}$ is bounded by some constant $c_{m, n}>0$ (as it is a continuous function of $\eta \in[-\pi, \pi]$ ) so that (100) and the triangle inequality give us

$$
\left(1+c_{m, n}^{\prime} h\right) \Lambda_{n}^{h} \leq h \mu_{m}+(1+|\ln h|) h\left(c_{m, n}+1\right) \leq c_{m, n}^{\prime \prime}(1+|\ln h|) h .
$$

Inserting this again to the right hand side of (100) yields $\Lambda_{n}^{h} \leq h \mu_{m}+O\left(h^{2}(1+\right.$ $\left.|\ln h|)^{2}\right)=O(h)$. Using this and (100) once more gives us

$$
\left|\Lambda_{n}^{h}-h \mu_{m}\right| \leq C_{n} h^{2}(1+|\ln h|),
$$

i.e. (98) holds true with $\mu_{m}$ in the place of $\mu_{n}$.

Remark 2 Proof of Lemma 1. We can now complete the proof for the formula (47) and thus also Lemma 1 . Indeed, fixing $m \in \mathbb{N}$, we have found, as a consequence of (101), for each eigenvalue $\mu_{k}, k \leq m$, with multiplicity $\varkappa_{k}$ at least $\varkappa_{k}$ eigenvalues $\Lambda_{J(k)}^{h}, \ldots, \Lambda_{J(k)+\varkappa_{k}-1}^{h}$ belonging to the the interval

$$
\left[h \mu_{k}-C_{k} h^{2}(1+|\ln h|), h \mu_{k}+C_{k} h^{2}(1+|\ln h|)\right]
$$

for $h \in\left(0, h^{(k)}\right)$. At this point we do not know, if there are eigenvalues outside the segments (102), nevertheless, we can conclude that, for $h<\min \left\{h^{(1)}, \ldots, h^{(m)}\right\}$, there are at least $m-1$ eigenvalues in the intervals (102) with $k=1, \ldots, m-1$. Thus, $J(m) \geq m$ and, hence,

$\Lambda_{m+j}^{h}(\eta) \leq \Lambda_{J(m)+j}^{h}(\eta) \leq h \mu_{m}+C_{m} h^{2}(1+|\ln h|) \leq C_{m}^{\prime} h, \quad j=0, \ldots, \varkappa_{m}-1$.

Lemma 6 Let $n \in \mathbb{N}$ and $\eta \in[0,2 \pi)$ be fixed and consider the eigenvalue $\mu_{n}=$ $\mu_{n}(\eta)$, see (45), and denote its multiplicity by $\mathcal{J}=\mathcal{J}(n, \eta) \in \mathbb{N}$. Then, for some $\hat{h}>0$, the interval

$$
\triangle_{n, \eta}=\left[h\left(\mu_{n}-C_{n}\right), h\left(\mu_{n}+C_{n}\right)\right],
$$

contains for all $h \in(0, \hat{h}]$ at least $\mathcal{J}$ eigenvalues $\Lambda_{p}^{h}(\eta)$ with multiplicities counted.

In (103), the number $C_{n}=C_{n}(\eta)$ is, say, half of the distance of $\mu_{n}(\eta)$ to the nearest different eigenvalue of the limit problem.

Proof. Given $\mathcal{J}$ orthonormalized eigenvectors $v_{p(n)+j}^{0}, j=1, \ldots, \mathcal{J}$, see (46), corresponding to $\mu_{n}$ we construct $\mathcal{J}$ approximate eigenvectors $\mathcal{W}_{p(n)+j}^{h}$ for all $j$ as in (64), (65) by using the vectors $v_{p(n)+j}^{0}$. Lemma 5 then shows that the vectors $\mathcal{W}_{p(n)+j}^{h}$, $j=1, \ldots, \mathcal{J}$ form a linearly independent sequence, if $h$ is small enough.

For all $j$, we take $\mathcal{U}_{p(n)+j}^{h}=\mathcal{W}_{p(n)+j}^{h}\left\|\mathcal{W}_{p(n)+j}^{h} ; H_{\eta}^{h}\right\|^{-1}$, see (69), and $\delta_{*}^{h}=h^{-3 / 4}$ in Lemma 2; note that the latter choice is possible, since $\delta_{*}^{h} \in\left(\delta^{h}, k^{h}\right)$ and $k^{h}$ is of order $h^{-1}$. Due to (70) and the estimate (97) in Corollary 1, the inequality (59) holds with $\delta^{h} / \delta_{*}^{h}=c h^{3 / 4}(1+|\ln h|)$ and with the true eigenvectors $U_{k(n)+j}^{h}, j=1, \ldots, \mathcal{X}^{h}$, see (20), of $\mathcal{K}_{\eta}^{h}$ in place of $u_{p}^{h}$, with the corresponding eigenvalues $\kappa_{k(n)+j}^{h}$, (17), 
belonging to the interval $\left[k_{n}-\delta_{*}^{h}, k_{n}+\delta_{*}^{h}\right]$. We denote for a moment by $\Lambda$ and $\kappa$ any of the eigenvalues $\Lambda_{k(n)+j}^{h}$ and $\kappa_{k(n)+j}^{h}, j=1, \ldots, \mathcal{X}^{h}$, respectively, and obtain by the relations (16), (63)

$$
h^{-3 / 4}=\delta_{*}^{h} \geq\left|\kappa^{h}-k_{n}^{h}\right|=\left|\frac{1}{\Lambda^{h}+h}-\frac{1}{h \mu_{n}+h}\right|=\frac{\left|\Lambda^{h}-h \mu\right|}{h\left(\Lambda^{h}+h\right)(\mu+1)} .
$$

Hence, since $\Lambda^{h} \leq$ ch by (47), we get

$$
\left|\Lambda^{h}-h \mu_{n}\right| \leq c h^{5 / 4}
$$

and this shows that the eigenvalues $\Lambda_{k(n)+j}^{h}, j=1, \ldots, \mathcal{X}^{h}$, belong to $\triangle_{n, \eta}$. According to (14), (59), we get for some numbers $b_{j}$,

$$
\begin{aligned}
& \left\|\mathcal{U}_{p(n)+j}^{h}-\sum_{j=1}^{\mathcal{X}^{h}} b_{j} U_{p(n)+j}^{h} ; L^{2}\left(\omega^{0}\right)\right\| \\
& \leq h^{-1 / 2}\left\|\mathcal{U}_{p(n)+j}^{h}-\sum_{j=1}^{\mathcal{X}^{h}} b_{j} U_{p(n)+j}^{h} ; \mathcal{H}_{\eta}^{h}\right\| \leq h^{-1 / 2} \frac{\delta^{h}}{\delta_{*}^{h}} \leq c h^{1 / 5} .
\end{aligned}
$$

In view of the normalization of the vectors $\mathcal{U}_{p(n)+j}^{h}$, multiplying (105) by $\| \mathcal{W}_{p(n)+j}^{h}$; $H_{\eta}^{h} \| \leq c h^{1 / 2}($ see (94)) also yields

$$
\left\|\mathcal{W}_{p(n)+j}^{h}-\sum_{j=1}^{\mathcal{X}^{h}} b_{j}^{\prime} U_{p(n)+j}^{h} ; L^{2}\left(\omega^{0}\right)\right\| \leq c h^{7 / 10}
$$

for some numbers $b_{j}^{\prime}$. Now, if $\mathcal{X}^{h}<\mathcal{J}$ and $h$ is small enough, we arrive at a contradiction, since, by (93) in Lemma 5, the $\mathcal{J}$ vectors $\mathcal{W}_{p(n)+j}^{h}$ form a nearly orthonormal sequence in $L^{2}\left(\omega^{0}\right)$ and the subspace spanned by the vectors $U_{p(n)+j}^{h}$ is only $\mathcal{X}^{h}$ dimensional.

Proof of Theorem 1. From Lemma 6 we actually obtain that given $n \in \mathbb{N}$, (101) holds for some $m \leq n$ : note that the entries in both sequences (19) and (45) are in an increasing order and that the intervals (103) are disjoint.

It thus suffices to show that $m \geq n$ in (101), and to this end it is enough to prove that in the notation of Remark 6, the case $\mathcal{J}<\mathcal{X}^{h}$ cannot happen; here, $\mathcal{J}$ is the multiplicity of $\mu_{n}$ and $\mathcal{X}^{h}$ is the total multiplicity of the eigenvalues $\Lambda_{p(j)}^{h}$ belonging to the interval $\triangle_{n, \eta},(103)$.

Suppose that for some $\widehat{h}>0$ we have $\mathcal{J}<\mathcal{X}^{h}$, for infinitely many $h \in(0, \widehat{h})$ forming a set with 0 as an accumulation point. Then, for each such $h$ have $\mathcal{X}^{h}$ eigenfunctions $U_{p(j)}^{h}, j=1, \ldots, \mathcal{X}^{h}$ which are orthogonal to each other in the norm of $L^{2}\left(\omega^{0}\right)$, see (20). According to Lemma 1 and its proof, and the choice of the interval 
$\triangle_{n, \eta}$ we find a sequence $\left\{h_{q}\right\}_{q=1}^{\infty}$ such that

$$
h_{q}^{-1} \Lambda_{p(j)}^{h_{q}}(\eta) \rightarrow \mu_{n}(\eta) \text { as } q \rightarrow+\infty
$$

for all $j=1, \ldots, \mathcal{X}^{h}$, and also the eigenfunctions $U_{p(j)}^{h}$ converge to some eigenfunctions $v_{p(j)}^{0}$ of the eigenvalue $\mu_{n}(\eta)$ in the norm of $L^{2}\left(\omega_{\sharp}^{0}\right)$, as $q \rightarrow+\infty$; see (54). This leads to the conclusion that the $\mathcal{X}^{h}$ limit functions $v_{p(j)}^{0}$ are also mutually orthogonal in $L^{2}\left(\omega_{\sharp}^{0}\right)$ so that they in particular are linearly independent. Thus, there exist at least $\mathcal{J}+1$, linearly independent eigenfunctions of the limit problem corresponding to $\mu_{n}$, which contradicts the choice of $\mathcal{J}$.

Now, the eigenvalues of both the model and limit problems are arranged into an increasing order, so the indices $m$ and $n$ must be the same in (101), i.e., (98) holds.

\section{Detecting spectral gaps}

\subsection{Limit problem with a new small parameter}

In order to analyse the possible existence of spectral gaps in the spectrum (20) of the Steklov problem (4)-(6), we consider the limit problem (25),(26),(28),(42) with the additional assumptions that

$$
H(y)=1, \quad \theta^{\varepsilon}=\left\{y: \zeta:=\varepsilon^{-1} y \in \theta^{1}\right\}
$$

where $\theta^{1}$ is a domain in $\mathbb{R}^{2}$ surrounded by a smooth simple closed contour $\partial \theta^{1}$ and $\varepsilon>0$ is a new small parameter. We denote by $\left\{v^{\varepsilon}(y), \mu^{\varepsilon}(\eta)\right\}$ an eigenpair of the limit problem in this special case. Sending $\varepsilon \rightarrow+0$, which means glueing the small hole, we end up with the following problem in the rectangle $\omega=(-1 / 2,1 / 2) \times(-\ell, \ell)$ :

$$
\begin{aligned}
& -\Delta_{y} v^{0}(\eta ; y) 0 \mu^{0}(\eta) v^{0}(\eta ; y), y \in \omega, \\
& \quad \pm \frac{\partial v^{0}}{\partial y_{2}}\left(\eta ; y_{1}, \pm \ell\right)=0,\left|y_{1}\right|<\frac{1}{2}, \\
& v^{0}\left(\eta ; \frac{1}{2}, y_{2}\right)=e^{i \eta} v^{0}\left(\eta ;-\frac{1}{2}, y_{2}\right),\left|y_{2}\right|<\ell \\
& \quad \frac{\partial v^{0}}{\partial y_{1}}\left(\eta ; \frac{1}{2}, y_{2}\right)=e^{i \eta} \frac{\partial v^{0}}{\partial y_{1}}\left(\eta ;-\frac{1}{2}, y_{2}\right),\left|y_{2}\right|<\ell,
\end{aligned}
$$

the solutions of which are given by the formulas

$$
\begin{aligned}
& \mu_{j k}^{0}(\eta)=(2 \pi j+\eta)^{2}+\frac{\pi^{2} k^{2}}{4 \ell^{2}}, \\
& v_{j k}^{0}(\eta ; y)=e^{i(2 \pi j+\eta) y_{1}} \cos \left(\frac{\pi k}{2 \ell}\left(y_{2}+\ell\right)\right), j \in \mathbb{Z}, k \in \mathbb{N}_{0}
\end{aligned}
$$


(a)

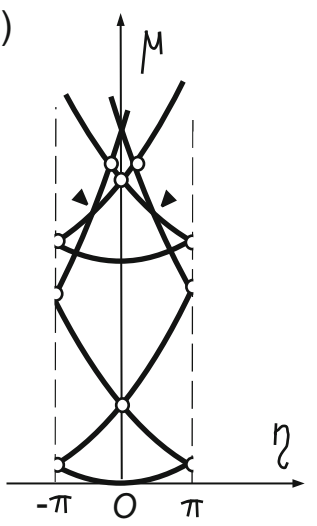

(b)

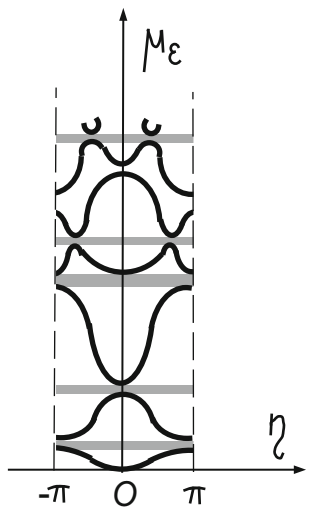

Fig. 4 a Dispersion curves, $\mathbf{b}$ opening of narrow gaps

The dispersion curves $\mu=\mu_{j k}^{0}(\eta), \eta \in[-\pi, \pi]$, form the grid in Fig. 4a), where the disposition of its knots is drawn in the case

$$
\frac{1}{6}<\ell<\frac{1}{4}
$$

We will consider the behavior of the dispersion curves of the above described singular perturbation problem and show that the knots, marked with $\circ$ in Fig. 4a), disintegrate and give rise to small spectral gaps of width $O\left(\varepsilon^{2}\right)$, which are presented by thick lines on the ordinate axis.

Asymptotic analysis as used here was initiated [20] and it has been applied in different spectral problems with singular and regular perturbation of boundaries, see $[3,6]$ and others. However, in view of non-standard integro-differential boundary conditions (42) on the small contour $\partial \theta^{\varepsilon}$, explicit formulas for the asymptotics of eigenvalues $\mu_{j k}^{\varepsilon}(\eta)$ would be required although error estimates would not be needed: the problem in $\omega \backslash \overline{\theta^{\varepsilon}}$ has a variational formulation, and the justification scheme follows word-byword those in $[3,6,20]$.

\subsection{Asymptotic ansätze}

We consider the perturbation of the knot $\left(0,4 \pi^{2}\right)$ as in Fig. $\left.4 a\right)$. The eigenvalue $\mu_{ \pm 10}(0)=4 \pi^{2}$ of the problem (107) has multiplicity two and eigenfunctions are

$$
v_{ \pm 10}(y)=e^{ \pm 2 \pi i y_{1}}
$$

Following $[3,20]$ we define the fast Floquet variable

$$
\psi=\varepsilon^{-2} \eta
$$


and introduce the asymptotic representation

$$
\mu^{\varepsilon}(\eta)=4 \pi^{2}+\varepsilon^{2} \mu^{\prime}(\psi)+\ldots
$$

The corresponding eigenfunctions are sought in the form

$$
v^{\varepsilon}(\eta ; y)=v^{0}(\psi ; y)+\varepsilon^{2} v^{2}(\psi ; u)+\varepsilon \chi_{\theta}(y)\left(w^{0}\left(\psi ; \varepsilon^{-1} y\right)+\varepsilon w^{1}\left(\psi ; \varepsilon^{-1} y\right)\right)+\ldots
$$

where $\chi_{\theta} \in C^{\infty}(\bar{\omega})$ is a cut-off function such that

$$
\begin{gathered}
\chi_{\theta}(y)=1, \text { if }\left|y_{1}\right|<1 / 6 \text { and }\left|y_{2}\right|<\ell / 3, \\
\chi_{\theta}(y)=0, \text { if }\left|y_{1}\right|>1 / 3 \text { or }\left|y_{2}\right|>2 \ell / 3,
\end{gathered}
$$

$v_{ \pm}^{p}$ are solutions of regular type in $\omega$ and $w^{q}$ are boundary layers written in the stretched coordinates $\zeta$, see (106), in the aperture domain $\Upsilon=\mathbb{R}^{2} \backslash \overline{\theta^{1}}$.

The first regular term consists of the linear combination

$$
v^{0}(\psi ; y)=A(\psi) e^{+2 \pi i y_{1}}+B(\psi) e^{-2 \pi i y_{1}}
$$

where the coefficients are to be determined. The second term $v_{ \pm}^{2}$ satisfies the equations

$$
\begin{aligned}
& -\Delta_{y} v^{2}(\psi ; y)-4 \pi^{2} v^{2}(\psi ; y)=\mu^{\prime}(\psi) v^{0}(\psi ; y)+f(\psi ; y), \quad y \in \omega, \\
& \frac{\partial v^{2}}{\partial y_{2}}\left(\psi ; y_{1},+\ell\right)-\frac{\partial v^{2}}{\partial y_{2}}\left(\psi ; y_{1},-\ell\right)=0, \quad\left|y_{a}\right|<\frac{1}{2},
\end{aligned}
$$

while, due to the relation $e^{i \eta}=e^{i \varepsilon^{2} \psi}=1+i \varepsilon^{2} \psi+O\left(\varepsilon^{4} \psi^{2}\right)$, the quasi-periodicity conditions become

$$
\begin{aligned}
& v^{2}\left(\psi ; \frac{1}{2}, y_{2}\right)=v^{2}\left(\psi ;-\frac{1}{2}, y_{2}\right)+i \psi v^{0}\left(\psi ;-\frac{1}{2}, y_{2}\right), \quad\left|y_{2}\right|<\ell \\
& \frac{\partial v^{2}}{\partial y_{1}}\left(\psi ; \frac{1}{2}, y_{2}\right)=\frac{\partial v^{2}}{\partial y_{1}}\left(\psi ;-\frac{1}{2}, y_{2}\right)+i \psi \frac{\partial v^{0}}{\partial y_{1}}\left(\psi ;-\frac{1}{2}, y_{2}\right), \quad\left|y_{2}\right|<\ell .
\end{aligned}
$$

The right-hand side $f$ will be determined in the next section after the examination of boundary layers. Then, the two compatibility conditions in problem (112)-(113) will give us the correction term in (109) as well as coefficients of the linear combination (110). 


\subsection{Boundary layer}

Stretching the coordinates $y \mapsto \zeta=\varepsilon^{-1} y$ and setting $\varepsilon=0$ turn the Eq. (25) with $H=1$ and (42) with $\theta=\theta^{\varepsilon}$ into

$$
\begin{aligned}
& -\Delta_{\zeta} w(\zeta)=0, \quad \zeta \in \mathbb{R}^{2} \backslash \overline{\theta^{1}}, \\
& w(\zeta)=a \in \mathbb{R}, \quad \zeta \in \partial \theta^{1}, \quad \int_{\partial \theta^{1}} \partial_{\nu} w(\zeta) d s_{\zeta}=0 .
\end{aligned}
$$

Indeed, we have

$$
\Delta_{y}+\mu^{\varepsilon}(\eta)=\varepsilon^{-2}\left(\Delta_{\zeta}+\varepsilon^{2} \mu^{\varepsilon}(\eta)\right), \quad \mu^{\varepsilon}(\eta) \operatorname{mes}_{2} \theta^{\varepsilon}=O\left(\varepsilon^{2}\right)
$$

and, therefore, in the limit $\varepsilon \rightarrow+0$ the Helmholtz operator becomes the Laplacian and the right hand side of the integral condition (42) vanishes.

We write the Taylor formula

$$
\begin{aligned}
& v^{0}(\psi ; y)=v^{0}(\psi ; 0)+y \cdot \nabla_{y} v^{0}(\psi ; 0)+O\left(|y|^{2}\right) \\
& =v^{2}(\psi ; 0)+\varepsilon \zeta \cdot \nabla_{y} v^{0}(\psi ; 0)+O\left(\varepsilon^{2}|\zeta|^{2}\right) .
\end{aligned}
$$

Since the constant $a$ is arbitrary, the first term $v^{0}(0)$ does not leave a discrepancy in the problem (114), (115). Furthermore,

$$
\int_{\partial \theta^{1}} \partial_{\nu} \zeta_{j} d s_{\zeta}=\int_{\partial \theta^{1}} v_{j}(\zeta) d s_{\zeta}=0, \quad j=1,2
$$

and therefore we need to find a harmonic function $\mathbf{w}$ satisfying

$$
\mathbf{w}_{j}(\zeta)=a_{j}-\zeta_{j}, \quad \zeta \in \partial \theta^{1}
$$

We recall the definition of the polarization matrix $P\left(\theta^{1}\right)$ [26, Appendix G], which is a $(2 \times 2)$-matrix (a tensor of rank 2$)$ and is composed from the coefficients in the decomposition of the decaying solution of the problem (114), (116),

$$
\mathbf{w}_{j}\left(\zeta_{j}\right)=\frac{1}{2 \pi} \sum_{k=1}^{2} P_{j k}\left(\theta^{1}\right) \frac{\zeta_{k}}{|\zeta|^{2}}+\widetilde{\mathbf{w}}_{j}(\zeta), \quad \widetilde{\mathbf{w}}_{j}(\zeta)=O\left(\frac{1}{|\zeta|^{2}}\right)
$$

The matrix $P\left(\theta^{1}\right)$ is symmetric and positive definite, see [26], and the last condition in (115) is fulfilled due to Green's formula

$$
\int_{\partial \theta^{1}} \partial_{\nu} \mathbf{w}_{j}(\zeta) d s_{\zeta}=-\lim _{R \rightarrow+\infty} \int_{\partial \mathbb{B}_{R}} \frac{\partial \mathbf{w}^{j}}{\partial|\zeta|}(\zeta) d s_{\zeta}=0
$$


and the decay rate $O\left(|\zeta|^{-2}\right.$ ) of the gradient $\nabla_{\zeta} \mathbf{w}_{j}(\zeta)$, see (117). Thus, this leads us to set

$$
w^{1}(\psi ; \zeta)=\sum_{k=1}^{2} \mathbf{w}_{j}(\zeta) \frac{\partial v^{0}}{\partial y_{j}}(\psi ; 0)
$$

According to [17], [16, Ch. 9,10] we also need to examine the next boundary layer term $w^{2}(\eta ; \zeta)$ which takes into account the second-order term

$$
Q(\psi ; y)=\frac{1}{2} \sum_{p, q=1}^{2} y_{p} y_{q} \frac{\partial^{2} v^{0}}{\partial y_{p} \partial y_{q}}(\psi ; 0)
$$

in the Taylor formula for $v^{0}$. Noting that $\left|\theta^{\varepsilon}\right|=\varepsilon^{2}\left|\theta^{1}\right|$ we get

$$
\int_{\partial \theta^{1}} \partial_{\nu} Q(\psi ; \zeta) d s_{\zeta}=\int_{\theta^{1}} \Delta_{\zeta} Q(\psi ; \zeta) d \zeta=\left|\theta^{1}\right| \Delta_{y} v^{0}(\psi ; 0)=-4 \pi^{2}\left|\theta^{1}\right| v^{0}(\psi ; 0) .
$$

Consequently, the functions $w^{2}$ must satisfy the Laplace equation as well as the conditions

$$
\begin{aligned}
& w^{2}(\psi ; \zeta)=a^{2}(\psi)-Q(\psi ; \zeta), \\
& \quad \int_{\partial \theta^{1}} \partial_{\nu} w^{2}(\psi ; \zeta ; \psi) d s_{\zeta}=4 \pi^{2}\left|\theta^{1}\right| v^{0}(\psi ; 0, \psi)=: T^{0}(\psi)
\end{aligned}
$$

and thus it can be decomposed as

$$
w^{2}(\psi ; \zeta)=T^{0}(\psi) \frac{1}{2 \pi} \ln \frac{1}{|\zeta|}+T^{1}(\psi ; \ln \varepsilon)+\widetilde{w}^{2}(\psi ; \zeta), \quad \widetilde{w}^{2}(\psi ; \zeta)=O\left(\frac{1}{|\zeta|}\right)
$$

We mention that the harmonic functions $w_{ \pm}^{2}$ grow at infinity and thus it is convenient to choose the constant

$$
T^{1}(\psi ; \ln \varepsilon)=T^{0}(\psi) \frac{1}{2 \pi} \ln \frac{1}{\varepsilon},
$$

which allows us to write

$$
\varepsilon^{2} w^{2}(\psi ; \zeta)=\varepsilon^{2} T^{0}(\psi) \frac{1}{2 \pi} \ln \frac{1}{|y|}+O\left(\frac{\varepsilon^{3}}{|y|}\right) .
$$

Finally, we take into account (118), (117) and obtain

$$
\varepsilon w^{1}(\psi ; \zeta)=\frac{\varepsilon^{2}}{2 \pi} \sum_{j, k=1}^{2} P_{j k}\left(\theta^{1}\right) \frac{y_{k}}{\left|y_{k}\right|} \frac{\partial v^{0}}{\partial y_{k}}(\psi ; 0)+O\left(\frac{\varepsilon^{3}}{|y|}\right) .
$$


Now we insert the ansatz (119) into the equation (25), separate terms of order $\varepsilon^{2}$, and taking into account (120), (121), complete the composition of the equation (112) by setting

$$
\begin{aligned}
& f(\psi ; y)=\left(\left[\Delta_{y}, \chi_{\theta}(y)\right]+\mu^{0}(\psi) \chi_{\theta}(y)\right) \mathcal{T}(\psi ; y), \text { where } \\
& \mathcal{T}(\psi ; y)=T^{0}(\psi) \frac{1}{2 \pi} \ln \frac{1}{|y|}+\sum_{j, k=1}^{2} P_{j k}\left(\theta^{1}\right) \frac{y_{k}}{\left|y_{k}\right|} \frac{\partial v^{0}}{\partial y_{k}}(\psi ; 0) .
\end{aligned}
$$

\subsection{Algebraic system for $\mu^{\prime}(\Psi)$}

Since $4 \pi^{2}$ is an eigenvalue of multiplicity 2 , the problem (112)-(113) must be associated with two compatibility conditions, which can be derived by inserting a possible solution $v_{ \pm}^{2}$ and the eigenfunctions (108) into Green's formula

$$
\begin{aligned}
& I_{ \pm}^{1}(\psi)+I_{ \pm}^{2}(\psi):=\mu_{ \pm}^{\prime}(\psi) \int_{\omega} \overline{v_{ \pm 10}(y)} v^{0}(\psi ; y) d y+\int_{\omega} \overline{v_{ \pm 10}(y)} f_{ \pm}(\psi ; y) d y \\
& \quad=\left.\int_{-\ell}^{\ell}\left(v^{2}(\psi ; y) \overline{\frac{\partial v_{ \pm 10}}{\partial y_{1}}(y)}-\overline{v_{ \pm 10}(y)} \frac{\partial v^{2}}{\partial y_{1}}(\psi ; y)\right)\right|_{y_{1}=-1 / 2} ^{y_{1}=1 / 2} d y_{2}=: I_{ \pm}^{3}(\psi) .
\end{aligned}
$$

Let us compute the integrals $I_{ \pm}^{p}(\psi)$. Owing to (108) and (110), we readily obtain

$$
I_{+}^{1}(\psi)=2 \ell A(\psi) \mu^{\prime}(\psi), \quad I_{-}^{1}(\psi)=2 \ell B(\psi) \mu^{\prime}(\psi)
$$

Using Green's formula in the perforated rectangle $\omega \backslash \mathbb{B}_{\delta}$ yields

$$
\begin{aligned}
& I_{ \pm}^{2}(\psi)=-\lim _{\delta \rightarrow+0} \int_{\omega \backslash \mathbb{B}_{\delta}} \overline{v_{ \pm 10}(y)}\left(\Delta_{y}+4 \pi^{2}\right)\left(\chi_{\delta}(y) \mathcal{T}(\psi ; y)\right) d y \\
& =\lim _{\delta \rightarrow+0} \int_{\omega \backslash \mathbb{B}_{\delta}}\left(\mathcal{T}(\psi ; y) \overline{\partial_{|y|} v_{ \pm 10}(y)}-\overline{v_{ \pm 10}(y)} \partial_{|y|} \mathcal{T}(\psi ; y)\right) d s_{y} \\
& =4 \pi^{2}\left(\left|\theta^{\prime}\right|(A(\psi)+B(\psi)) \pm P_{11}\left(\theta^{\prime}\right)((A(\psi)-B(\psi))\right.
\end{aligned}
$$

Recalling the relations (113) gives us

$$
\begin{gathered}
I_{ \pm}^{3}(\psi)=4 \pi \ell \psi((A(\psi)+B(\psi))+((A(\psi)-B(\psi))) \\
\Rightarrow I_{+}^{3}(\psi)=8 \pi \ell \psi A(\psi), \quad I_{-}^{3}(\psi)=-8 \pi \ell \psi A(\psi) .
\end{gathered}
$$


(a)

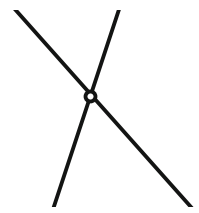

(b)

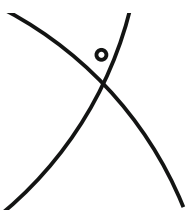

(c)

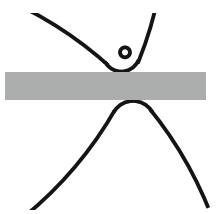

Fig. 5 a, b Knot of the dispersion curves, $\mathbf{c}$ disintegrating knot

Combining formulas (122)-(123) we arrive at the system of linear algebraic equations

$$
\begin{gathered}
\left(\mathcal{P}_{+}\left(\theta^{1}\right)-8 \pi \ell \psi\right) A(\psi)+\mathcal{P}_{-}\left(\theta^{1}\right) B(\psi)=-2 \ell A(\psi) \mu^{\prime}(\psi), \\
\mathcal{P}_{-}\left(\theta^{1}\right) A(\psi)+\left(\mathcal{P}_{+}\left(\theta^{1}\right)+8 \pi \ell \psi\right) B(\psi)=-2 \ell B(\psi) \mu^{\prime}(\psi),
\end{gathered}
$$

where

$$
\mathcal{P}_{ \pm}\left(\theta^{1}\right)=4 \pi^{2}\left(\left|\theta^{1}\right| \pm P_{11}\left(\theta^{1}\right)\right)
$$

Thus,

$$
\mu_{ \pm}^{\prime}(\psi)=-\frac{1}{2 \ell}\left(\mathcal{P}_{ \pm}\left(\theta^{1}\right) \pm \sqrt{\mathcal{P}_{ \pm}\left(\theta^{1}\right)^{2}+64 \pi^{2} \ell^{2} \psi^{2}}\right) .
$$

\subsection{Conclusions on spectral gaps}

If $\mathcal{P}_{-}\left(\theta^{1}\right)=0$, the graphs of the functions $\psi \mapsto \mu_{ \pm}^{\prime}(\psi)$ include two crossing straight lines (Fig. 5a), but in the case

$$
\mathcal{P}_{-}\left(\theta^{1}\right) \neq 0
$$

the graphs turn into two disjoint parabolas (Fig. 5c). Thus, in the same way as in the papers cited above, we conclude that the knot $\left(4 \pi^{2}, 0\right)$ in Fig. $4 \mathrm{~b}$ disintegrates as in Fig. 4c, and causes a spectral gap of width $\left.\ell^{-1} \varepsilon^{2} \mathcal{P}_{-}\left(\theta^{1}\right)\right)+O\left(\varepsilon^{3}\right)$ with the center at the point $4 \pi^{2}-(2 \ell)^{-1} \varepsilon^{2} \mathcal{P}_{+}\left(\theta^{1}\right)+O\left(\varepsilon^{3}\right)$.

To give an example where (124) holds, we observe that for a horizontal crack $\overline{\theta^{1}}=\left\{\zeta: \zeta_{2}=0,\left|\zeta_{1}\right|<L\right\}$ we have

$$
\left|\theta^{\prime}\right|=0 \text { and } P_{11}\left(\theta^{\prime}\right)=\pi L^{2}>0,
$$

see [26, Appendix G].

An example of a smooth domain with (124) is given by observing that for a thin ellipse $\theta_{\delta}^{1}$ with axes 1 and $\delta$ we have (see again [26, Appendix G])

$$
\left|\theta_{\delta}^{1}\right|=O(\delta) \text { and } P_{11}\left(\theta_{\delta}^{1}\right)=\pi^{2}\left(1+O\left(\delta^{2}\right)\right) / 4>0 .
$$


The equality $\mathcal{P}_{-}(\theta)=0$ does not yet imply the non-existence of a gap, but this depends on higher order asymptotic terms.

Similar calculations can be applied to study the existence of gaps near all knots marked with $\circ$ in Fig. 4b. On the other hand, the knots marked with •, which are obtained as the intersection of either two ascending or two descending curves, never get disjoined (see an explanation in, e.g., [20]).

Funding Open Access funding provided by University of Helsinki including Helsinki University Central Hospital.

\section{Declarations}

Conflict of interest The authors declare that they have no conflict of interest.

Open Access This article is licensed under a Creative Commons Attribution 4.0 International License, which permits use, sharing, adaptation, distribution and reproduction in any medium or format, as long as you give appropriate credit to the original author(s) and the source, provide a link to the Creative Commons licence, and indicate if changes were made. The images or other third party material in this article are included in the article's Creative Commons licence, unless indicated otherwise in a credit line to the material. If material is not included in the article's Creative Commons licence and your intended use is not permitted by statutory regulation or exceeds the permitted use, you will need to obtain permission directly from the copyright holder. To view a copy of this licence, visit http://creativecommons.org/licenses/by/4.0/.

\section{References}

1. Agmon, S., Douglis, A., Nirenberg, L.: Estimates near the boundary for solutions of elliptic differential equations satisfying general boundary conditions. Comm. Pure Appl. Math. (4) 12, 623-727 (1959)

2. Agmon, S., Douglis, A., Nirenberg, L.: Estimates near the boundary for solutions of elliptic differential equations satisfying general boundary conditions. Comm. Pure Appl. Math. (1) 17, 35-92 (1964)

3. Bakharev, F.L., Nazarov, S.A., Ruotsalainen, K.M.: A gap in the spectrum of the Neumann-Laplacian on a periodic waveguide. Appl. Anal. 88, 1889-1995 (2012)

4. Bakharev, F.L., Pérez, M.-E.: Spectral gaps for the Dirichlet-Laplacian in a 3-D waveguide periodically perturbed by a family of concentrated masses. Math. Nachr. 291(4), 556-575 (2018)

5. Birman, M.S., Solomyak, M.Z.: Spectral Theory of Self-Adjoint Operators in Hilbert Space. Reidel Publishing Company, Dordrecht (1986)

6. Borisov, D.I., Pankrashkin, K.V.: Quantum waveguides with small periodic perturbations: gaps and edges of Brillouin zones. J. Phys. A: Math. Theor. 46, 235203 (2013)

7. Cancedda, A., Chiadó Piat, V., Nazarov, S. A., Taskinen, J.: Spectral gaps for the linear water-wave problem in a channel with thin structures. Submitted

8. Cardone, G., Durante, T., Nazarov, S.A.: Water-waves modes trapped in a canal by a near-surface rough body. Zeitschrift Angew. Math. Mechan. ZAMM 90, 983-1004 (2010)

9. Gelfand, I.M.: Expansion in characteristic functions of an equation with periodic coefficients. Dokl. Akad. Nauk SSSR 73, 1117-1120 (1950). ((in Russian))

10. Ilin, V.A.: Matching of asymptotic expansions of solutions of boundary-value problems. Nauka, Moscow (1989). English transl.: Transl. Math. Monogr. 102. Amer. Math. Soc., Providence, RI (1992)

11. Kato, T.: Perturbation theory for linear operators. Die Grundlehren der mathematischen Wissenschaften, Band 132 Springer-Verlag, New York, (1966)

12. Kondratiev, V.A.: Boundary-value problems for elliptic equations in domains with conical or angular points. Trudy Moskov. Matem. Obshch. 16, 209-292 (1967). English transl.: Trans. Moscow Math. Soc. 16, 227-313 (1967)

13. Kuchment, P.: Floquet Theory for Partial Differential Equations. Birkhäuser, Basel (1993)

14. Kuznetsov, N., Maz'ya, V.G., Vainberg, B.: Linear Water Waves: A Mathematical Approach. Cambridge University Press, Cambridge (2002) 
15. Lions, J.-L., Magenes, E.: Non-homogeneous boundary value problems and applications, Vol. I. Die Grundlehren der mathematischen Wissenschaften, Band 181, Springer-Verlag, New York-Heidelberg (1972).

16. Mazya, V.G., Nazarov, S.A., Plamenevskii, B.A.: Asymptotic Theory of Elliptic Boundary Value Problems in Singularly Perturbed Domains. Birkhäuser Verlag, Basel (2000)

17. Maz'ya, V.G., Nazarov S.A., Plamenevskii, B.A.: Asymptotic expansions of the eigenvalues of boundary value problems for the Laplace operator in domains with small holes. Izv. Akad. Nauk SSSR. Ser. Mat . 48, 347-371 (1984). English transl.: Math. USSR Izvestiya 24, 321-345 (1985)

18. Nazarov, S.A.: Properties of spectra of boundary value problems in cylindrical and quasicylindrical domains. In: Maz'ya, V.G. (ed.) Sobolev Spaces in Mathematics, vol. II International Mathematical Series 9, 261-309 (2008)

19. Nazarov, S.A.: Asymptotic conditions at a point, self-adjoint extensions of operators and the method of matched asymptotic expansions. Trudy St.-Petersburg Mat. Obshch. 5, 112-183 (1996). English transl.: Trans. Am. Math. Soc. Ser. 2. 193, 77-126 (1999)

20. Nazarov, S.A.: Opening a gap in the continuous spectrum of a periodically perturbed waveguide. Mat. Zametki. 87, 764-786 (2010). English transl.: Math. Notes. 87, 738-756 (2010)

21. Nazarov, S.A.: Asymptotic behavior of the eigenvalues of the Steklov problem on a junction of domains of different limiting dimensions. Comput. Math. Math. Phys. 52, 1574-1589 (2012)

22. Nazarov, S.A.: Modeling of a singularly perturbed spectral problem by means of self-adjoint extensions of the operators of the limit problems. Funkt. Anal. i Prilozhen. 49, 31-48 (2015). English transl.: Funct. Anal. Appl. 49, 25-39 (2015)

23. Nazarov, S.A., Orive-Illera, R., Péréz-Martínez, M.-E.: Asymptotic structure of the spectrum in a Dirichlet-strip with double periodic perforations. Netw. Heterogenous Media 14, 733-757 (2019)

24. Nazarov, S.A., Plamenevskii, B.A.: Elliptic Problems in Domains with Piecewise Smooth Boundaries. Walter be Gruyter, Berlin, New York (1994)

25. Nazarov, S.A., Taskinen, J.: On essential and continuous spectra of the linearized water-wave problem in a finite pond. Math. Scand. 106, 141-160 (2010)

26. Pólya, G., Szegö, G.: Isoperimetric inequalities in mathematical physics. Annals of Mathematics Studies 27, Princeton (1951)

27. Reed, M., Simon, B.: Methods of Modern Mathematical Physics, vol. IV. Academic Press, Cambridge (1978)

28. Van Dyke, M.: Perturbation methods in fluid mechanics. Appl. Math. Mech., vol. 8, Academic Press, New York-London (1964)

29. Visik, M.I., Ljusternik, L.A.: Regular degeneration and boundary layer of linear differential equations with small parameter. Amer. Math. Soc. Transl. 20, 239-364 (1962)

Publisher's Note Springer Nature remains neutral with regard to jurisdictional claims in published maps and institutional affiliations. 